\title{
Fatigue of double-network hydrogels
}

\section{Citation}

Zhang, Wenlei, Xiao Liu, Jikun Wang, Jingda Tang, Jian Hu, Tongqing Lu, and Zhigang Suo. 2018. “Fatigue of Double-Network Hydrogels." Engineering Fracture Mechanics 187 (January): 74-93. doi:10.1016/j.engfracmech.2017.10.018.

\section{Published Version}

doi:10.1016/j.engfracmech.2017.10.018

\section{Permanent link}

http://nrs.harvard.edu/urn-3:HUL.InstRepos:34902467

\section{Terms of Use}

This article was downloaded from Harvard University's DASH repository, and is made available under the terms and conditions applicable to Open Access Policy Articles, as set forth at http:// nrs.harvard.edu/urn-3:HUL.InstRepos:dash.current.terms-of-use\#OAP

\section{Share Your Story}

The Harvard community has made this article openly available.

Please share how this access benefits you. Submit a story.

\section{Accessibility}


To appear in a special issue that celebrates the 5oth anniversary of Engineering Fracture Mechanics

\title{
Fatigue of Double-Network Hydrogels
}

Wenlei Zhang ${ }^{1}$, Xiao Liu ${ }^{1}$, Jikun Wang ${ }^{1}$, Jingda Tang ${ }^{1}$, Jian $\mathrm{Hu}^{1}$, Tongqing $\mathrm{Lu}^{* 1}$, Zhigang Suo*2

${ }^{1}$ State Key Lab for Strength and Vibration of Mechanical Structures, International Center for Applied Mechanics, Department of Engineering Mechanics, Xi'an Jiaotong University, Xi'an 710049, China

${ }^{2}$ School of Engineering and Applied Sciences, Kavli Institute for Bionano Science and Technology, Harvard University, MA 02138

\begin{abstract}
The discovery of tough hydrogels of many chemical compositions, and their emerging applications in medicine, clothing, and engineering, has raised a fundamental question: How do hydrogels behave under many cycles of stretch? This paper initiates the study of the fatigue behavior of the classic PAMPS/PAAM double network hydrogels discovered by Gong and her co-workers (Advanced Materials 15, 1155, 2003). We reproduce the hydrogels, and prepare samples of two types, with or without a crack cut before the test. When an uncut sample is subject to cyclic stretches, internal damage accumulates over thousands of cycles until a steady state is reached. When a cut sample is subject to cyclic stretches, the crack extends cycle by cycle if the amplitude of stretch is above a certain value. A threshold of energy release rate exists, below which the crack remains stationary as the sample is cycled. We find a threshold around $400 \mathrm{~J} / \mathrm{m}^{2}$ for hydrogels containing PAAM networks of a low density of crosslinkers, and around $200 \mathrm{~J} / \mathrm{m}^{2}$ for hydrogels containing PAAM networks of a high density of crosslinkers. The experimental findings are compared to the Lake-Thomas model adapted to the double-network hydrogels.

keywords: double-network hydrogel, fatigue fracture, threshold, shakedown

*E-mail: tongqinglu@mail.xjtu.edu.cn; suo@seas.harvard.edu
\end{abstract}




\section{Introduction}

A hydrogel is an aggregate of water and a three-dimensional polymer network (Fig. 1). That is, the hydrogel is a molecular composite: polymer-reinforced water. The mesh of the polymer network is on the order of $10 \mathrm{~nm}$, much larger than an individual water molecule. Consequently, water in the hydrogel retains its molecular properties. In particular, water in the hydrogel is a solvent of small molecules, and transports them. The polymer network gives the hydrogel elasticity. The hydrogel is compliant under a mechanical force, and recovers its shape after the force is removed. Most tissues of animals and plants are hydrogels. Many synthetic hydrogels are compatible with living tissues chemically, mechanically, and electrically.

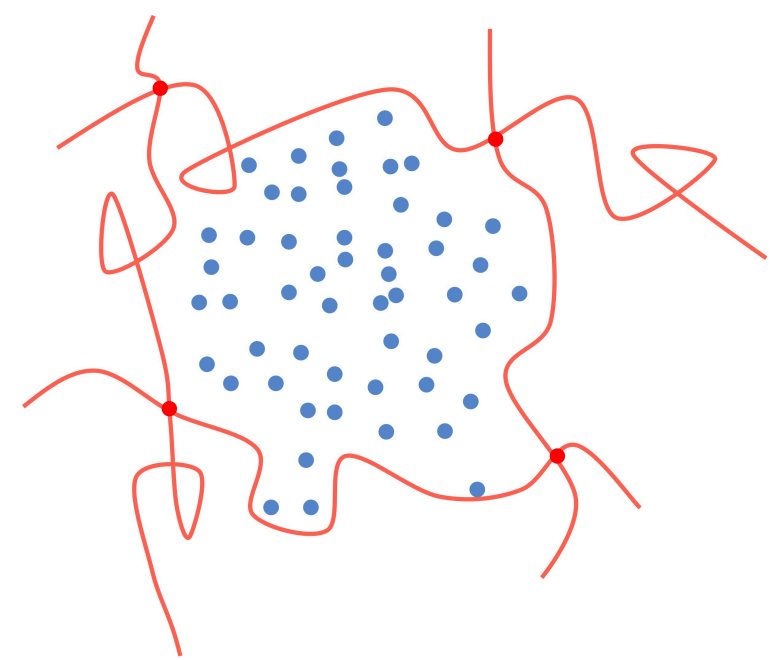

Fig. 1 A hydrogel is an aggregate of water and a polymer network. The mesh of the polymer network is much larger than an individual water molecule, so that water retains its molecular properties. 
The history of synthetic hydrogels is rather short [1]. Initial commercial successes were contact lenses in the 1960s [2] and superabsorbent diapers in the 1980s [3]. Recent decades have seen intense development for medical applications, such as drug delivery [4] and tissue regeneration [5]. When a hydrogel is immersed in water, the polymer network imbibes or exudes water in response to changes in force, temperature, voltage, light, and concentrations of molecular species. The large, environment-responsive swelling and de-swelling have been used to develop sensors and actuators [6, 7]. Hydrogels are stretchable, transparent, ionic conductors, and have enabled many devices, such as transparent loudspeakers [8], muscle-like actuators [8, 9], skin-like sensors [10, 11, 12], axon-like cables [13], stretchable touchpads $[14,15]$, stretchable electroluminescent displays [16, 17], transparent triboelectric generators [18], and stretchable liquid crystal devices [19]. Dissolved humectants and elastomeric coatings can retard evaporation of water and enable the devices to be used in open air [20-23]. These developments open doors for hydrogels to be used in smart textiles [23, 24]

Hydrogels are often brittle, like jello and tofu. The poor mechanical properties limit the scope of their applications. The situation has fundamentally changed since Gong and her co-workers discovered double-network (DN) hydrogels [25]. A DN hydrogel has two interpenetrating polymer networks (Fig. 2). A short-chain network, poly(1-acrylanmido-2-methylpropanesulfonic acid) (PAMPS), swells nearly to the breaking point. A long-chain network, polyacrylamide (PAAM), is highly stretchable. When the DN hydrogel is stretched, the short-chain network ruptures 
and dissipates energy, and the long-chain network retains elasticity. Thus, even with high water content (ca. 90wt\%), the DN hydrogels have outstanding stiffness (0.1-1MPa), strength (1-10MPa), and toughness $\left(100 \sim 1000 \mathrm{~J} / \mathrm{m}^{2}\right)$ [26]. The mechanical properties can be changed by varying the ratio of the monomers of the two networks and the densities of their crosslinkers [27].


Fig. 2 A double-network hydrogel consists of two polymer networks: a short-chain network swells nearly to the breaking point, and a long-chain network is highly stretchable. When the hydrogel is stretched, the short-chain network ruptures, but the long-chain network remains elastic.

The PAMPS/PAAM DN hydrogel has stimulated the research on the toughening mechanisms of hydrogels [28-37], as well as the discovery of tough hydrogels of many other chemical compositions [38-46]. For tough hydrogels having two interpenetrating networks, the two networks can be both chemically crosslinked, both physically crosslinked, or one chemically crosslinked and the other physically $10 / 21 / 2017$ 
crosslinked. Reported attributes include high toughness, high strength, large stretchability, and self-recovery [41, 47]. For example, alginate/polyacrylamide hydrogels achieve toughness above $10,000 \mathrm{~J} / \mathrm{m}^{2}$, comparable to that of natural rubber $[38,48]$. Tough hydrogels have also led to strong adhesion between hydrogels and other materials [49]. Moreover, tough hydrogels have been used as matrices to develop composites [51-53]. We call these composites water matrix composites (WMCs), in which water is the matrix, and the reinforcements are in two scales, as individual molecular chains and macroscopic fibers and particles. WMCs are, of course, matters of life-tissues of plants and animals.

A new question is being asked, what can we do when water is a tough solid? The discovery of tough hydrogels has stimulated efforts to seek applications in which high toughness plays essential roles. Examples include artificial cartilages [63-66], tissue adhesives [67], drug delivery [68], fire-retarding blankets [69], and soft robots $[70,71]$.

Tough materials derive their high toughness from large stress-stretch hysteresis (Fig. 3). This basic principle of fracture mechanics has long been established for metals [54,56], elastomers [56, 57], transformation-toughened ceramics [58], ceramic-matrix composites [59], and elastomer-toughened plastics [6o]. In the DN hydrogel, the hysteresis is due to the rupture of the short-chain network [26, 61, 62]. 
(a)

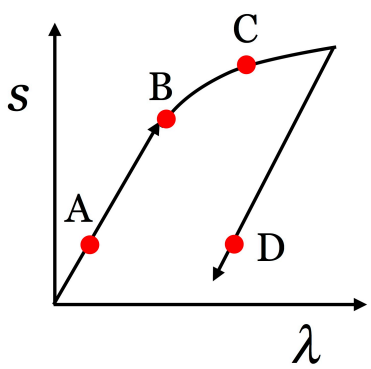

(b)

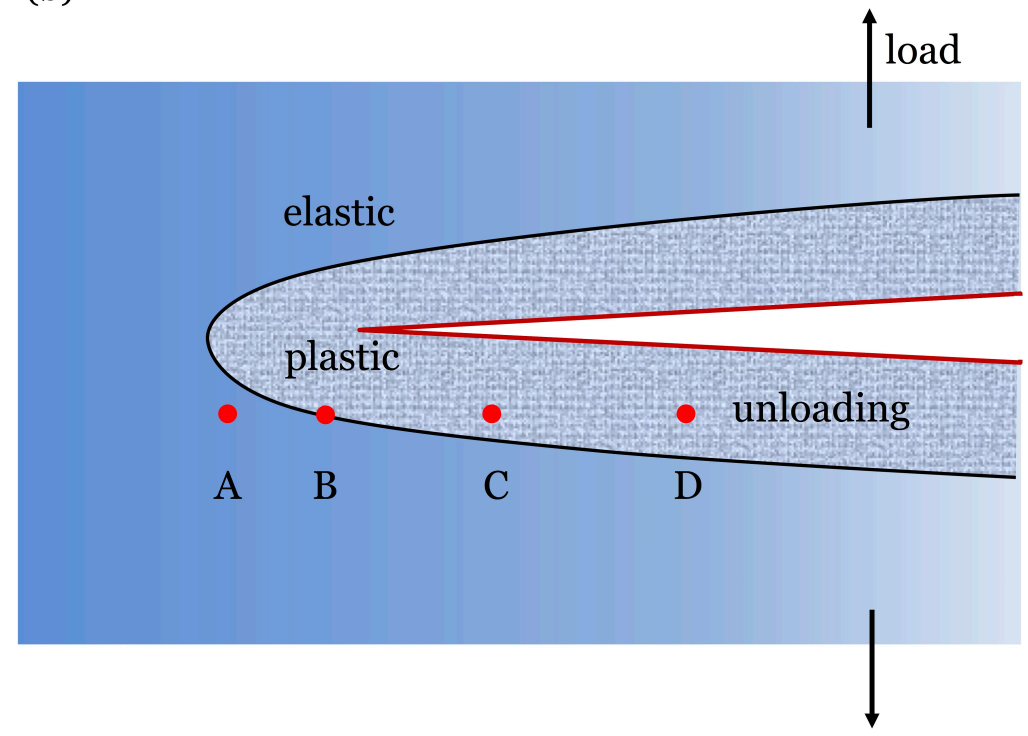

Fig. 3 A fundamental principle of fracture mechanics: hysteresis and toughness. (a)

When a material is subject to a cycle of loading and unloading, the stress-stretch curve exhibits a loop of hysteresis. (b) When the material with a preexisting crack is subject to a monotonic load, the crack extends, leaving behind two layers of the material going through hysteresis. The four material particles A, B, C, D correspond to the four states marked in the stress-stretch plane. The fracture energy equals the work done to create the two surfaces of the crack and the hysteresis in the two layers.

In the schematic of the stress-stretch curve (Fig. 3a), the elastic modulus during unloading is the same as the elastic modulus during loading, and a residual stretch is left after the stress reduces to zero. Both features are observed in metals, but need not be true for other materials. In particular, for a PAMPS/PAAM hydrogel, a large stretch breaks the PAMPS network, but leaves the PAAM network intact, so that the hydrogel has no residual stretch upon unloading to zero stress. Furthermore, the elastic modulus during unloading is lower than the elastic modulus during loading. 
In load-bearing applications, hydrogels are often subject to cyclic stretches. The hysteresis is much smaller in subsequent cycles than in the initial cycle [72]. That the DN hydrogels are prone to fatigue fracture often comes up in conversations, but has not been studied. Indeed, fatigue fracture of hydrogels of any kind has not been reported until recently. Three likely reasons for this lack of interest have been suggested [73]. First, synthetic hydrogels are a relatively new type of materials, and interest in their mechanical behavior started only after the commercialization of contact lenses in the 1960 s and superabsorbent diapers in the 1980 s. Second, fatigue fracture was not "mission-critical" in the initial applications of hydrogels. Third, hydrogels in use may degrade or dehydrate before they rupture under cyclic load.

The discovery of tough hydrogels, as well as their emerging applications in medicine, clothing, and engineering, strongly indicates that hydrogels will be used in applications that require many cycles of loads. Two sets of data on fatigue fracture of hydrogels have just appeared in the literature, one set for the polyacrylamide single-network hydrogel [73], and the other set for the alginate/polyacrylamide hydrogel [74] (Fig. 4). The threshold for fatigue fracture in the alginate/polyacrylamide hydrogel is much below the fracture energy under a monotonic load. Nonetheless, the extension of crack per cycle in the alginate/polyacrylamide hydrogel is much smaller than that in a single-network polyacrylamide hydrogel. The available data of fatigue fracture in the alginate/polyacrylamide hydrogel is obtained using samples prepared with particular 
ratios of water, polymers, and crosslinkers. Given that fracture energy is sensitive to these ratios, it is important to study their effects on fatigue behavior.

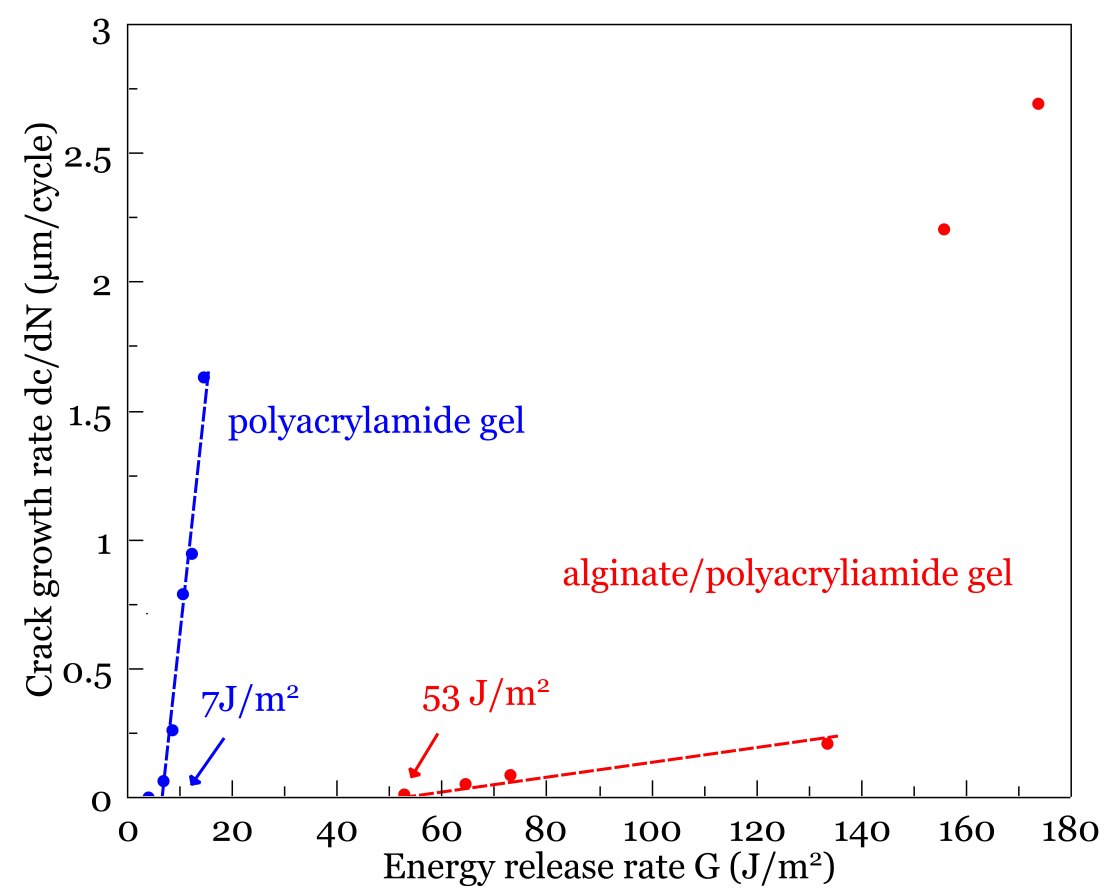

Fig. 4 The extension of crack per cycle as a function of the energy release rate for a polyacrylamide hydrogel and a particular composition of alginate/polyacrylamide hydrogel. Adapted from Refs [73] and [74].

Incidentally, the phrase "fatigue damage" designates degradation under cyclic load. Examples include cycle-by-cycle change in elastic modulus [75], in hysteresis of stress-stretch curves [43, 76], and in functional characteristics of devices [77]. By contrast, the phrase "fatigue fracture" designates rupture under cyclic load.

Here we initiate a study of the fatigue fracture of the PAMPS/PAAM double-network hydrogel discovered by Gong and her co-workers [25]. As noted above, this DN hydrogel launched the world-wide search for tough hydrogels and their applications. Furthermore, the DN hydrogel has two polymer networks of 
chemical crosslinks, and most other tough hydrogels involve networks of physical crosslinks. This difference may become significant in applications. The mechanical behavior of the DN hydrogel is affected by the concentrations of the monomers, crosslinkers, and initiators in the precursors. A comprehensive study of the effects of all these parameters is beyond the scope of this paper. Here we prepare three groups of samples in aqueous solutions of different AAM concentrations, and keep all other parameters fixed. We measure the stress-stretch curves using the uniaxial tensile test, and measure the toughness by using the pure shear test. We apply cyclic loads on uncut samples to observe the shakedown of stress-stretch curves. We apply cyclic loads on cut samples to observe the propagation of fatigue cracks. We compare the experimental findings to the Lake-Thomas model. We further synthesize a DN hydrogel with shorter PAAM chains, and find that the threshold of fatigue fracture is reduced.

\section{Synthesis of double-network hydrogels}

We synthesized DN hydrogels using the method of sequential formation of the two networks developed by Gong and her co-workers [25]. The ingredients were obtained from sources different from those for the DN gels reported in [25]. To achieve similar stress-stretch curves as in [25], we have altered the concentrations of the ingredients.

We purchased from Aladdin the following substances: 1-acrylanmido-2-methylpropanesulfonic acid (AMPS, monomer), acrylamide (AAM, 
monomer), N,N'-methylenebis (acrylamide) (MBAA, crosslinker) and 2-oxoglutaric acid (OA, initiator).

We prepared an aqueous solution of 1 M AMPS, and then added to the solution 4 mol\% MBAA and 0.1 mol\% OA relative to AMPS. The aqueous solution was injected into an acrylic mold of $10 \times 10 \times 0.5 \mathrm{~mm}^{3}$ and covered with a glass plate. The PAMPS network was formed under an ultraviolet lamp of $15 \mathrm{~W}$ and $365 \mathrm{~nm}$ for 8 hours in the environment full of argon gas.

We then immersed the PAMPS hydrogel in an aqueous solution of $2 \mathrm{M}, 3 \mathrm{M}$, or 4 M AAM to make it freely swell. Each solution contains 0.001mol\% MBAA and 0.005 mol\% OA relative to AAM. The PAMPS network is a polyelectrolyte, and swells nearly to the breaking point. After the immersion for at least one day, the piece of swollen hydrogel was sandwiched between a pair of glass plates, and placed in the same reaction environment as in the first step for 8 hours to form the PAAM network.

We finally immersed the DN hydrogels in the deionized water for 3 days to remove the residual unreacted substances, and to equilibrate the hydrogels into the fully swollen state. The average thickness in the fully swollen state was $1.3 \mathrm{~mm}$ for the hydrogels prepared using the solution of 2M AAM, $1.4 \mathrm{~mm}$ for $3 \mathrm{M} \mathrm{AAM}$, and $1.5 \mathrm{~mm}$ for 4M AAM.

We determined the volume fraction of the PAAM network in a DN hydrogel as follows. We prepared two identical PAMPS gels. We dehydrated one PAMPS gel at $60^{\circ}$ $\mathrm{C}$ for $12 \mathrm{~h}$ and measured the dried mass of PAMPS network $m_{P A M P S}$. We used the 
other PAMPS gel to prepare a DN hydrogel. After preparing the PAMPS/PAAM gel, we measured the mass and volume of the gel, $m_{g e l}$ and $V_{g e l}$. Finally, we dehydrated the PAMPS/PAAM gel at the same condition and measured the dried mass of two networks $m_{\text {polymer }}$. The volume fractions of the two networks in the final hydrogel, $\phi_{P A M P S}$ and $\phi_{P A A M}$, are calculated as

$$
\begin{gathered}
\phi_{P A M P S}=\frac{m_{P A M P S}}{\rho_{A M P S} V_{g e l}} \\
\phi_{P A A M}=\frac{\left(m_{\text {polymer }}-m_{P A M P S}\right)}{\rho_{A A M} V_{g e l}}
\end{gathered}
$$

The volume fractions $\phi_{P A M P S}$ and $\phi_{P A A M}$ are 1.2 and 7.4 vol\% for the DN hydrogel prepared using the solution of 2M AAM, 1.0 and 9.9 vol\% for $3 \mathrm{M} \mathrm{AAM}$, and 0.9 and 11.8 vol\% for 4M AAM. They correspond to water contents of 91.4, 89.1, and 87.3 vol\%, or 88,86 , and 84 wt\%.

\section{Uniaxial tensile test}

We used the as-prepared hydrogels for tensile tests. The hydrogels were cut using a razor blade into dumbbell-shaped samples with effective length $12 \mathrm{~mm}$ and width $2 \mathrm{~mm}$ (GB/T 528-2009-4 standard) (Fig. 5a). The samples were clamped using the two metallic grippers of a tensile tester (SHIMADZU AGS-X). The grippers were displaced by a pneumatic power source (JINGYIN QD1212). We used a load cell of $1000 \mathrm{~N}$ to apply a uniaxial monotonic load to each sample at a rate of $100 \mathrm{~mm} / \mathrm{min}$ until the sample ruptured. The tests were carried out at $25^{\circ} \mathrm{C}$ in the open air. Before rupture, the sample elongated significantly (Fig. 5b). The force and displacement for each sample were recorded by the tensile tester. We plotted the stress-stretch curves 
of all the samples (Fig. 5c, d, e). The nominal stress is the applied force in the deformed state divided by the cross-sectional area in the undeformed state. The stretch is the length in the deformed state divided by the length in the undeformed state. For each samples prepared in an aqueous solution of a given AAM concentration, the deviation of stress at a given stretch is within $20 \%$ compared to the average value.

Each stress-stretch curve terminates when the sample ruptures. Some samples rupture at the middle, but others rupture near the gripped ends. The latter are possibly due to stress concentration, and typically result in smaller rupture stretches. The tensile strengths and the rupture stretches of the DN hydrogel synthesized by us are comparable to those reported from the Gong Group [27]. The hydrogels prepared using the solution of $2 \mathrm{M}$ AAM demonstrate the highest yield stress and rupture after yield. The hydrogels prepared using the solution of 3 M AAM yield at a lower stress but a larger stretch in average. The hydrogels prepared using the solution of $4 \mathrm{M}$ AAM show pronounced strain stiffening, with the highest strength and the rupture stretch.

The shear modulus of each sample is obtained by fitting the beginning portion of its stress-stretch curve to the neo-Hookean model [78]:

$$
s=\mu\left(\lambda-\lambda^{-2}\right)
$$

where $s$ is the nominal stress, $\lambda$ is the stretch, and $\mu$ is the shear modulus. The shear modulus for all hydrogels prepared in this work is roughly $100 \mathrm{kPa}$ (See 
Appendix for fitting). As expected, the double-network hydrogels do not follow the neo-Hookean model. Our intention here is to obtain a rough value for the small-strain shear modulus for the materials prepared. This shear modulus will not be used in any of the following calculations.

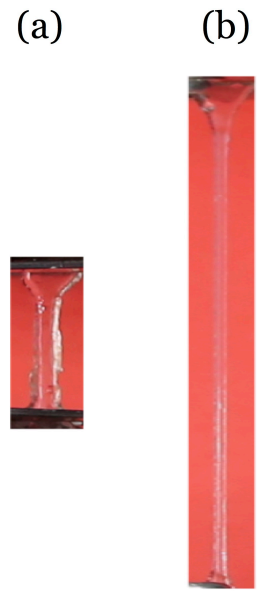

(d)

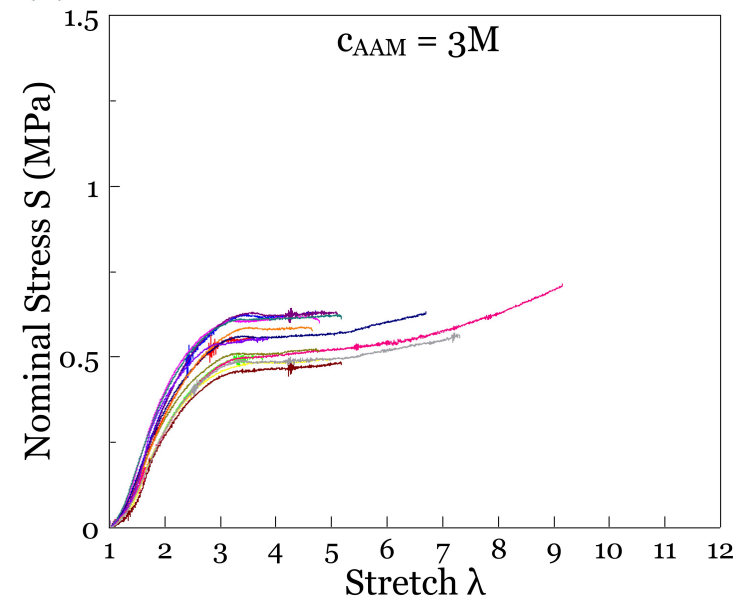

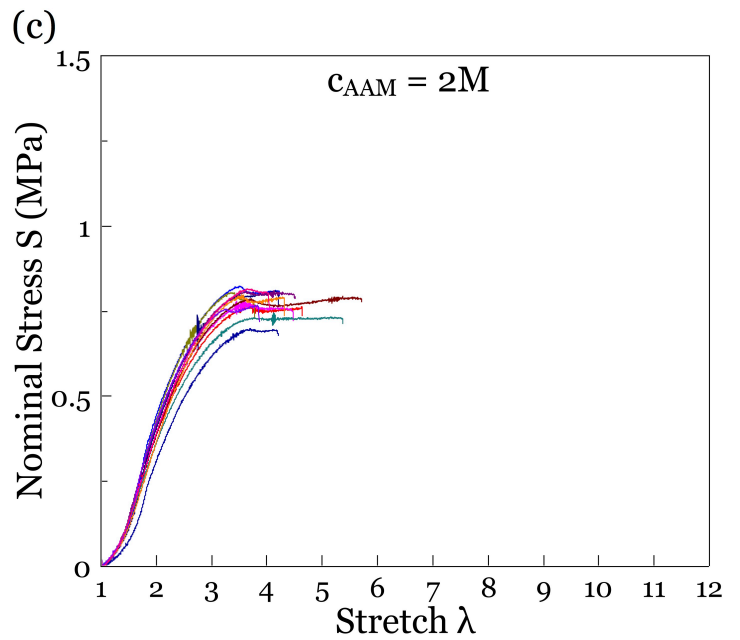

(e)

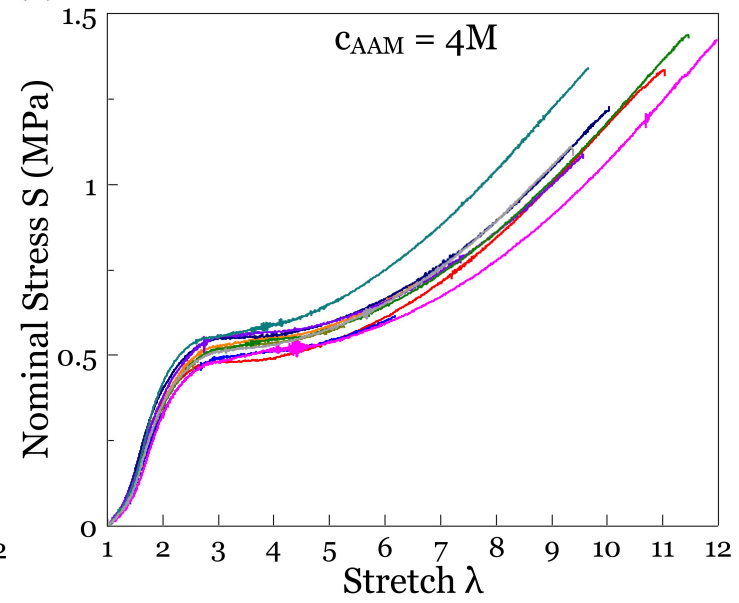

Fig. 5 Uniaxial tensile tests. (a), (b) A dumbbell-shaped sample, of 4 M AAM, in the undeformed state and a deformed state. (c), (d) and (e) Stress-stretch curves of samples prepared using the aqueous solutions of three AAM concentrations. Each curve terminates by the rupture of the sample.

\section{Fracture energy}


To measure toughness (also called tearing energy and fracture energy) of the PAMPS/PAAM hydrogels, we adopt an established method for rubber, the pure shear test [79]. Following [38], we prepared two sets of samples with the same geometry. One set is uncut, and the other set is precut with a crack.

The pure shear test usually takes several minutes for static fracture and at least 1 day for fatigue fracture. Thus, we sealed the samples in a homemade acrylic chamber (Fig. 6a). During the tests, we sprayed droplets of deionized water on the inner surface of the chamber. The difference between the weight of samples before and after testing was less than $5 \%$ of the initial weight.

The uncut sample was of a long rectangular sheet $(10 \mathrm{~mm} \times 50 \mathrm{~mm})$ and was clamped to the two grippers of the tensile tester (Fig. 6b). A monotonic load was applied at a rate of $30 \mathrm{~mm} / \mathrm{min}$. In the undeformed state, the height of the sample is $H$. In the deformed state, the height of the sample becomes $\lambda H$, where $\lambda$ is the applied stretch. The horizontal deformation was constrained by the rigid grippers. We plotted the stress-stretch curves of the uncut samples with three AAM concentrations in Fig. 6d-f.

The cut sample with the same geometry was prepared. A 20-mm crack was cut at the edge by using a razor blade. We applied the same load as that for the uncut samples and observed the opening and propagation of the crack (Fig. 6c). The critical stretches $\lambda_{C}$ when the cracks start to propagate are 2.35, 2.25, and 2.07 for samples prepared in solutions of different AAM concentrations. For both cut and uncut samples, 3 measurements were repeated for each AAM concentration and the average 
results are plotted in Fig. 6g-i. The deviation is within $10 \%$.

(a)

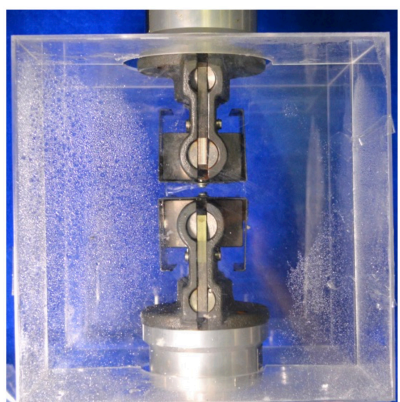

(d)

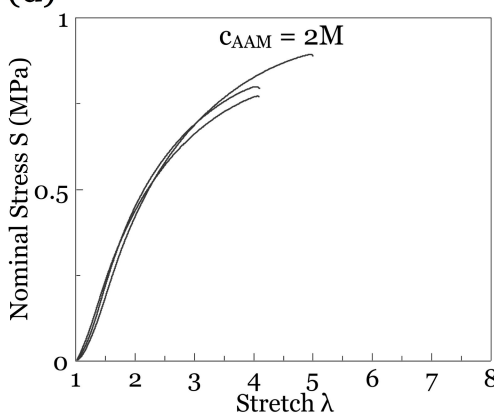

(g)

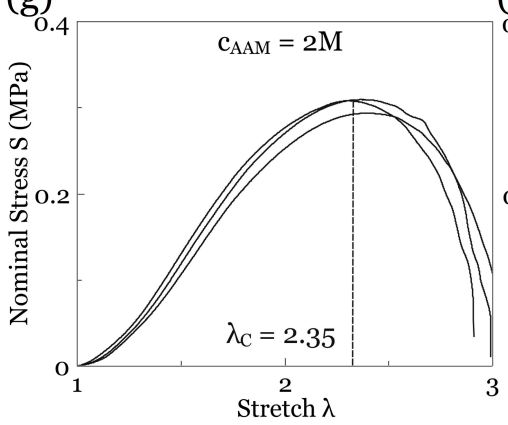

(b)
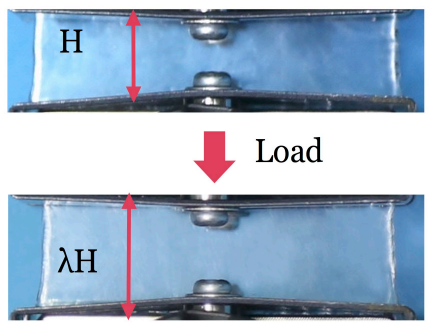

(e)

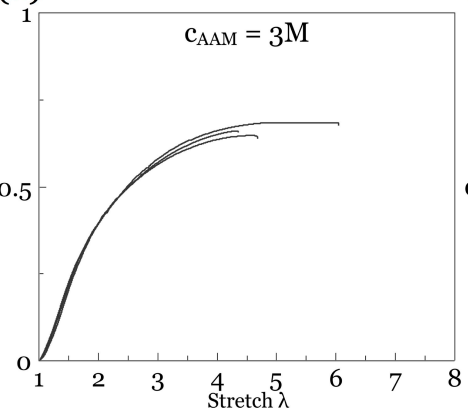

(h)

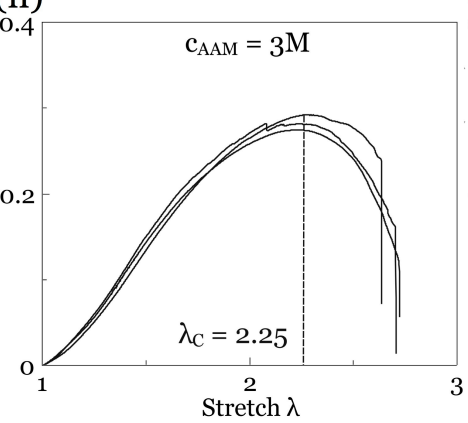

(c)

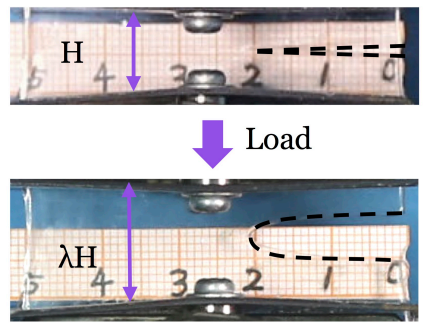

(f)

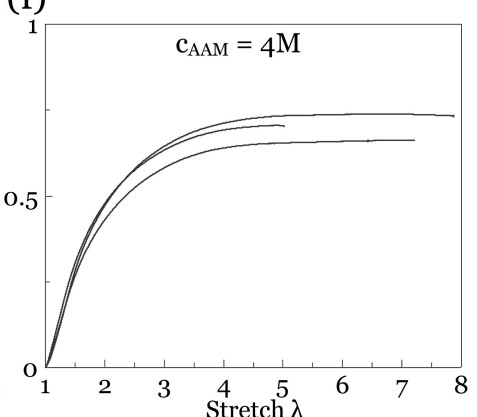

(i)

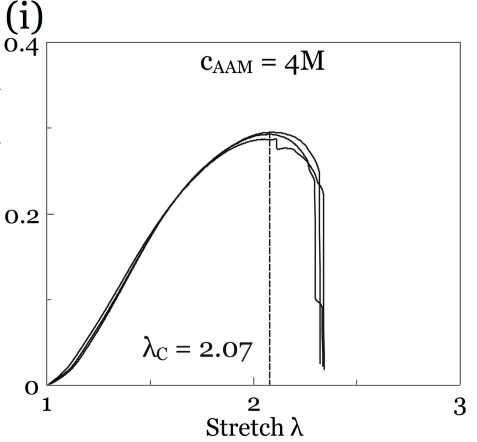

Fig. 6 Fracture test. (a) A sample is sealed in a humidity chamber, with water droplets sprayed in the interior walls of the chamber. (b) An uncut sample is subject to a monotonic load to a stretch $\lambda$. (c) A cut sample under a monotonic load. (d)-(f) The stress-stretch curves of the uncut samples of the PAMPS/PAAM hydrogels using solutions of different AAM concentrations. (g)-(i) The stress-stretch curves of the cut samples. $\lambda_{C}$ is the stretch of the cut samples when the crack begins to propagate. 
For the pure shear test, the stress-stretch relation with the neo-Hookean model is given by

$$
s=\mu\left(\lambda-\lambda^{-3}\right)
$$

Fitting the beginning portion of the curves in Fig. 6(d)-(f) gives the shear modulus of all the hydrogels roughly $100 \mathrm{kPa}$ (See Appendix for fitting).

For the pure shear test, the energy release rate takes the form [79].

$$
G=H W(\lambda)
$$

where $H$ is the distance between the two grippers when the sample is undeformed, $W(\lambda)$ is the energy per volume of the uncut samples, and $\lambda$ is the vertical stretch. The energy density $W(\lambda)$ is obtained by integrating the area below the stress-stretch curves of the uncut samples (Fig. 7a).

The toughness (i.e., the fracture energy) is defined as the critical energy release rate when the crack propagates. The toughness is calculated by replacing the stretch $\lambda$ in $W(\lambda)$ with the critical stretch of the cut samples, $\lambda_{C}$. The calculated toughness of the hydrogels prepared using the solutions of different AAM concentrations are shown in Fig. 7b. Each error bar shows the three repeated measurements. By comparison, the toughness of single-network polyacrylamide hydrogel is about $50 \mathrm{~J} / \mathrm{m}^{2}$ [73], and the toughness of natural rubber is about $10,000 \mathrm{~J} / \mathrm{m}^{2}[74]$ 
(a)

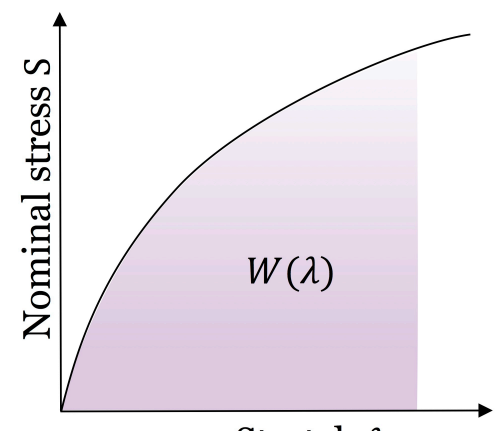

Stretch $\lambda$ (b)

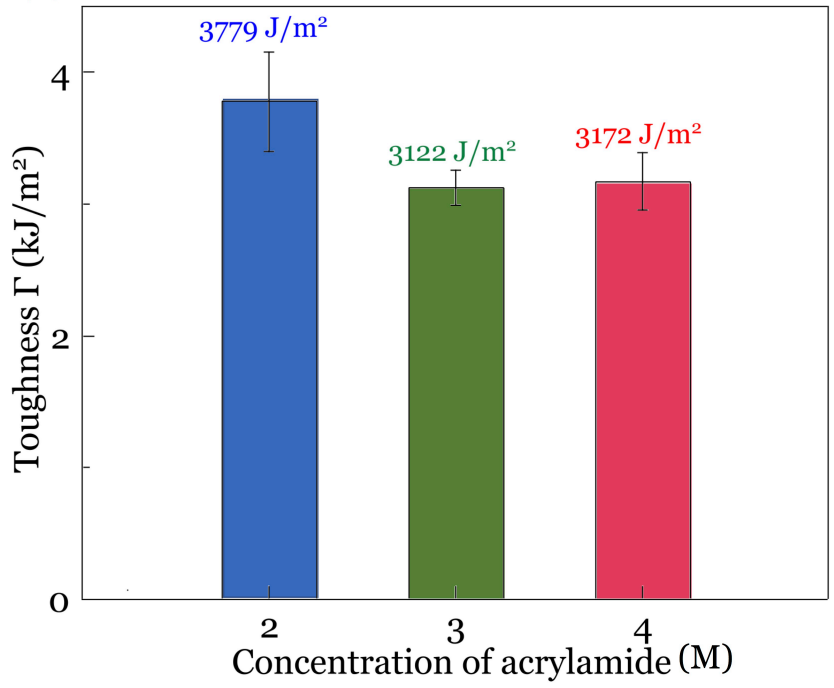

Fig. 7 Facture toughness. (a) The stress-stretch curve of an uncut sample measured in the pure shear test is integrated to obtain the energy density as a function of stretch, $W(\lambda)$. (b) Toughness of the PAMPS/PAAM double-network hydrogels prepared using the solutions of different AAM concentrations.

\section{Fatigue damage of uncut samples}

The stress-stretch curve of a DN hydrogel degrades under cyclic load because the short-chain network partially ruptures [72]. We now test how the state of rupture evolves over a large number of cycles. Using the pure shear setup, we applied cyclic loads to the uncut samples with a triangular loading profile of a frequency $0.4 \mathrm{~Hz}$. The minimum load and mean load may affect the fatigue properties of the DN hydrogel. Such effects have been studied for the fatigue fracture of elastomers [8o]. Here we set the loads cycle between $\lambda_{M I N}=1$ and a maximum stretch $\lambda_{\text {MAX }}$ for simplicity (Fig. 8a). 
The stress-stretch curve of the PAMPS/PAAM DN hydrogel lowers cycle by cycle (Fig. 8). Apparent residual stretch is found for each cycle when we unload the sample to zero stress. When we further unload the sample to $\lambda_{M I N}=1$, the sample suffers compressive stress and buckles. The parts of stress-stretch curve with negative stress are not shown in Fig.8. The maximum stress at the imposed stretch keeps decreasing over cycles. The hysteresis in the first cycle is much larger than the subsequent cycles. The change of the stress-stretch loops is negligible after 2000 cycles, and we say that the sample has shaken down to a steady state. By comparison, the polyacrylamide hydrogel shows little hysteresis, and the stress-stretch curves remain nearly unchanged cycle by cycle [73]. The alginate/polyacrylamide hydrogel shows even more significant hysteresis and shakedown than the PAMPS/PAAM hydrogel [74]. 
(a)

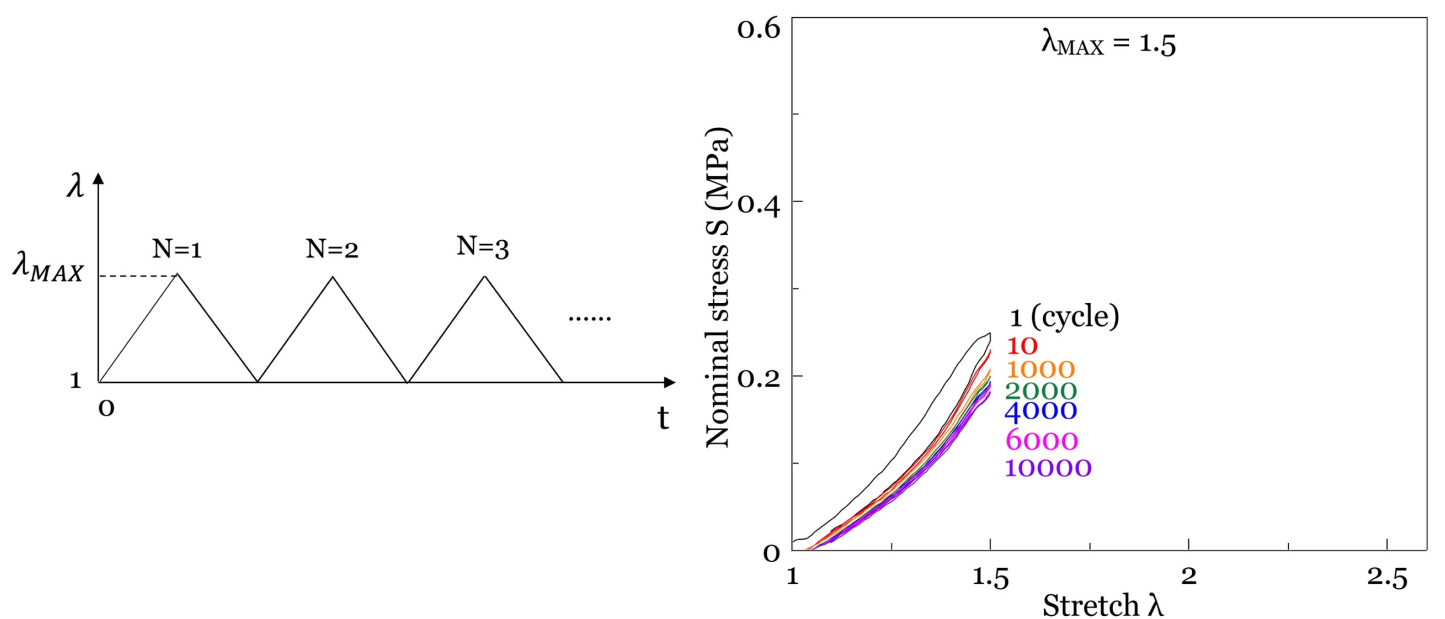

(c)

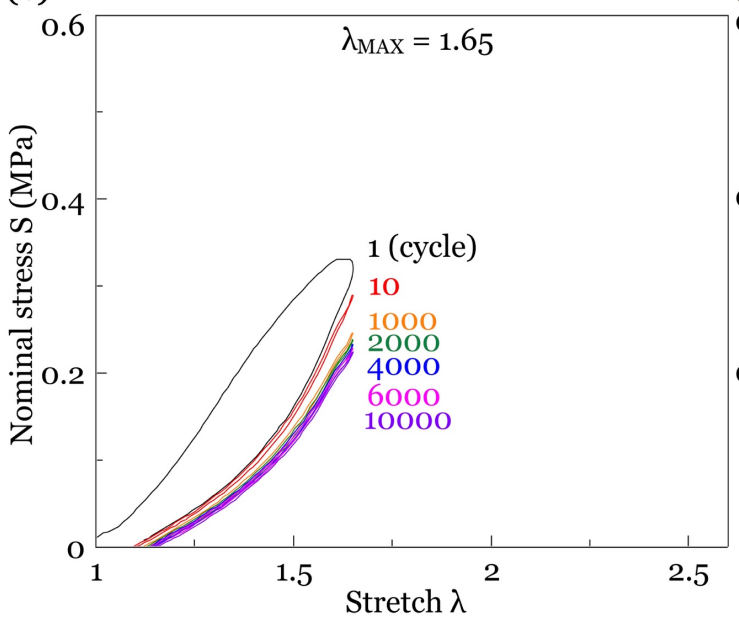

(e)

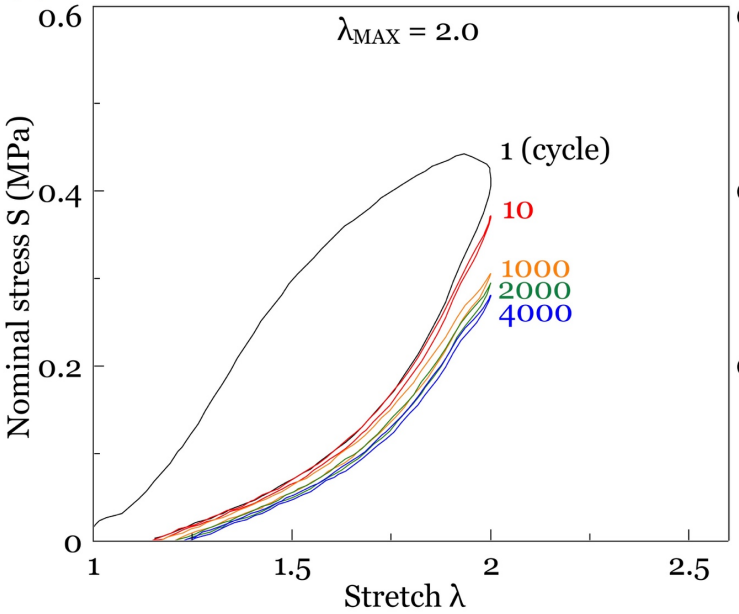

(b)

(d)

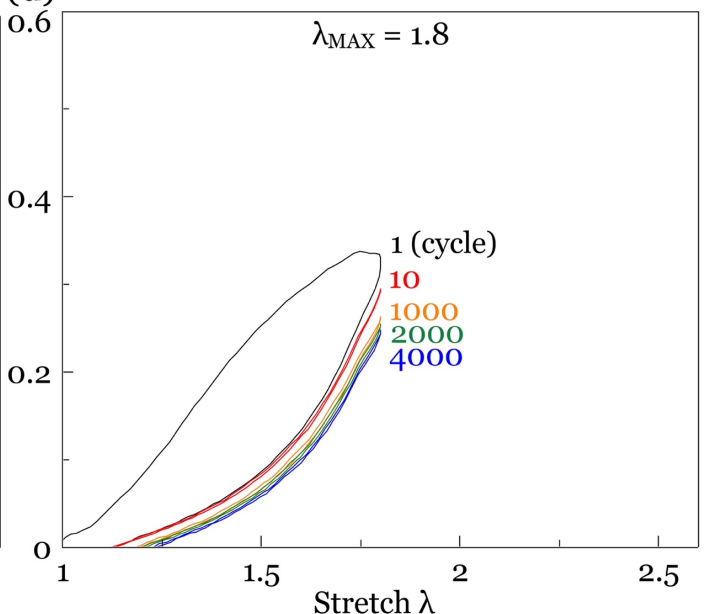

(f)

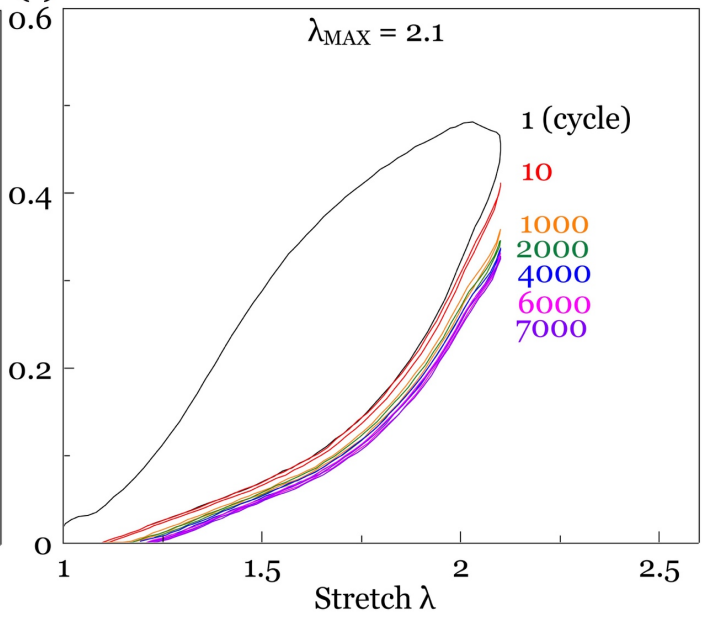

Fig. 8 Uncut samples shake down over thousands of cycles. The samples are prepared using the solution of $4 \mathrm{M}$ AAM. (See Appendix for the data for samples prepared using the solutions of $2 \mathrm{M}$ AAM and $3 \mathrm{M}$ AAM.) 
The shakedown phenomenon has been observed and studied in elastic-plastic materials, such as metals [83-89]. The shakedown of hydrogels over a large number cycles was reported for the alginate/polyacrylamide hydrogel [74], and now for the PAMPS/PAAM hydrogel. For the PAMPS/PAAM hydrogel, the first polymer network PAMPS is near the breaking point. Under cyclic loads, the PAMPS network partially ruptures into small clusters. The damage of the PAMPS network leads to the hysteresis between the loading and unloading curves. Our data suggests that each subsequent cycle incrementally involves more rupture of the PAMPS network, until a steady-state is reached.

The maximum stress drops greatly in the beginning cycles and reaches a steady state after thousands of cycles (Fig. 9a-c). Following [74], we use the stress-stretch curve of the $\mathbf{2 0 0 0}^{\text {th }}$ cycle to represent the deformation behavior of the PAMPS/PAAM hydrogels under cyclic load in the steady state (Fig. 9d-f). 
(a)

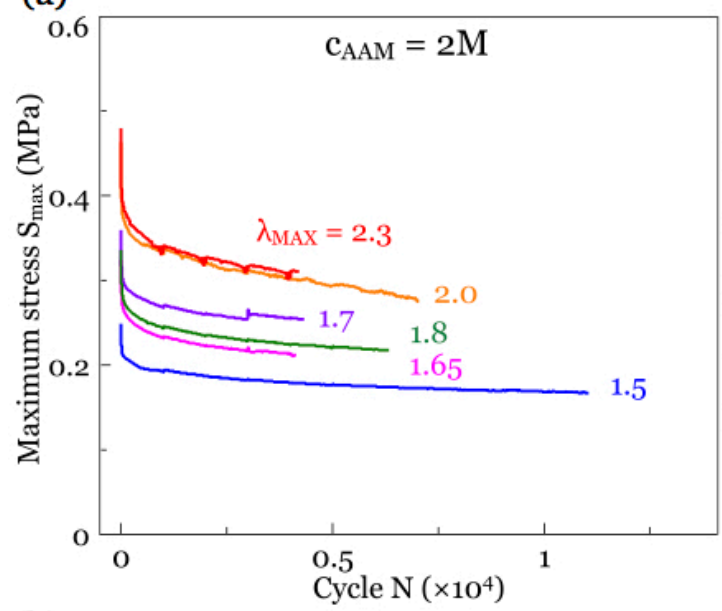

(b)

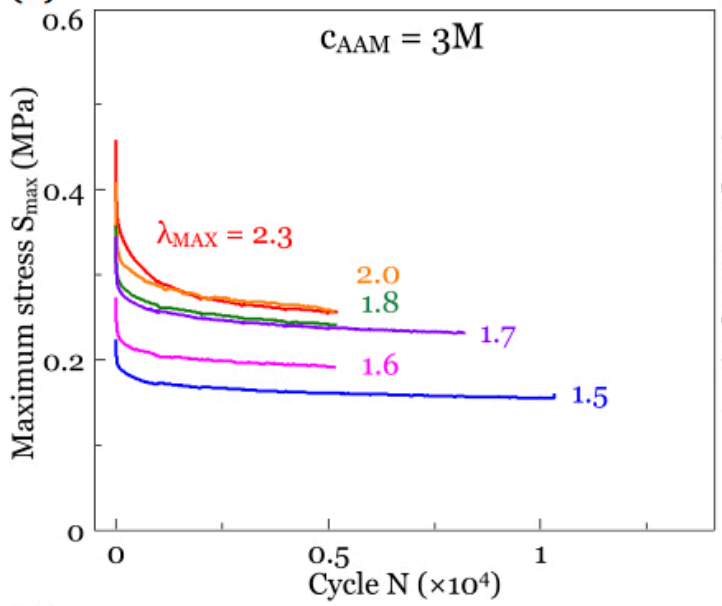

(c)

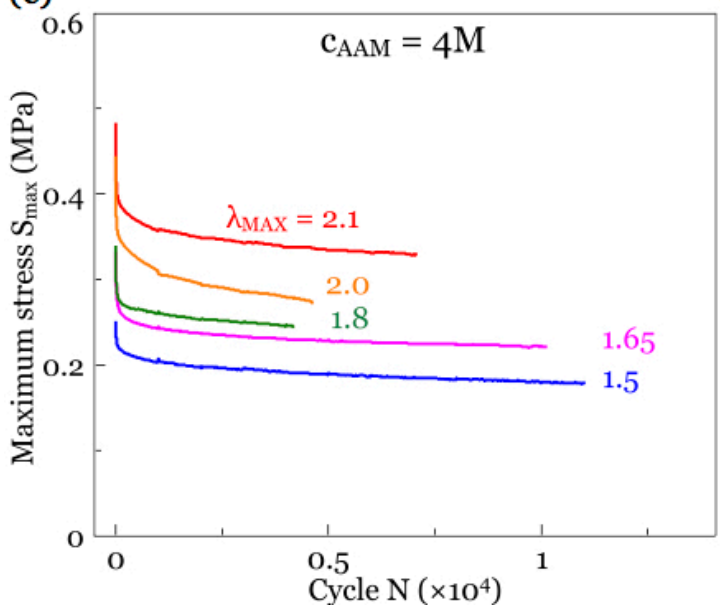

(d)

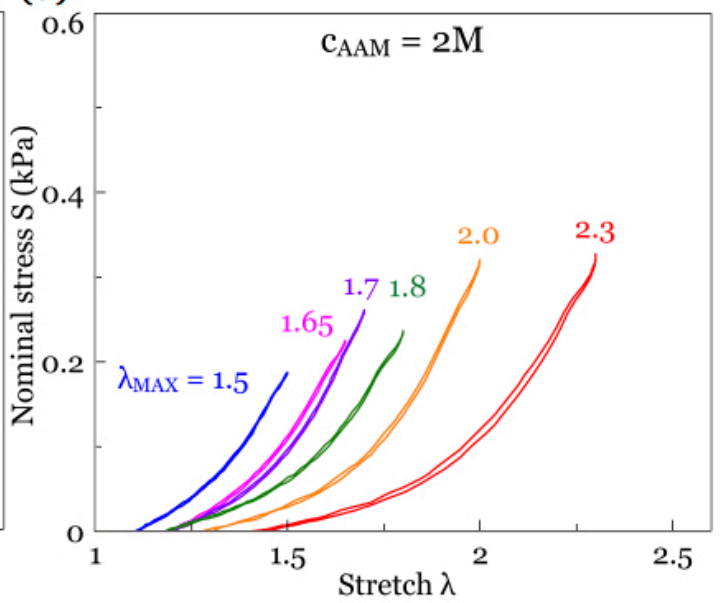

(e)

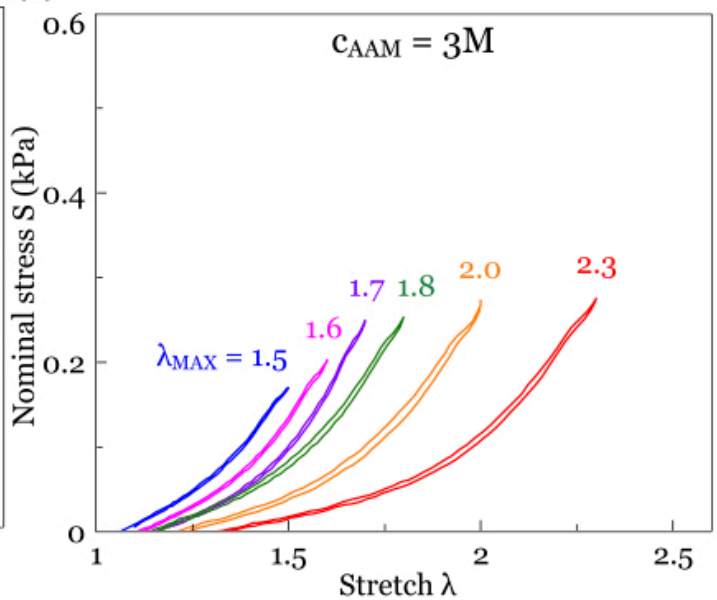

(f)

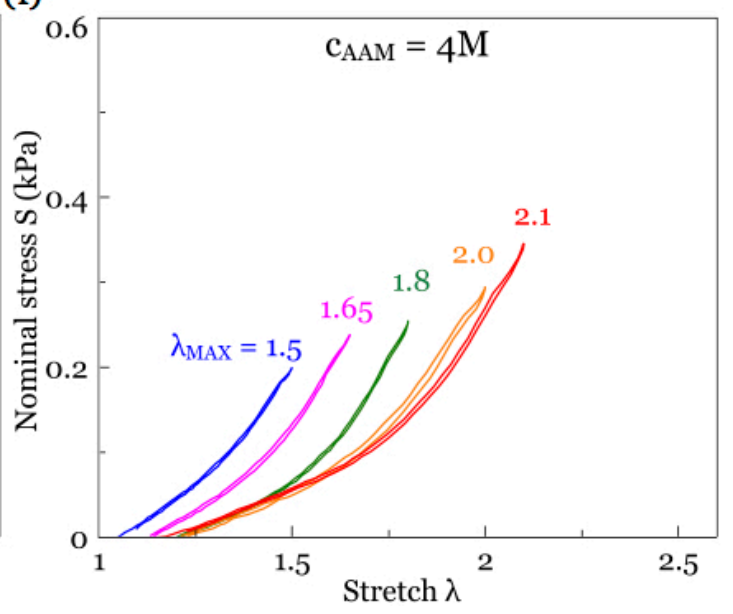

Fig. 9 Shakedown and the steady state. (a)-(c) The maximum stress of the stress-stretch curve is a function of the number of cycles, plotted for samples tested using different maximum loading stretches $\lambda_{M A X}$. (d)-(f) The stress-stretch curves of the $2000^{\text {th }}$ cycle. 


\section{Fatigue facture of cut samples}

To study the fatigue fracture of the DN hydrogels, we made a thin sheet of the same rectangular shape $(10 \mathrm{~mm} \times 50 \mathrm{~mm})$, and cut a $20-\mathrm{mm}$ crack using a razor blade. The cyclic loads with different maximum stretches $\lambda_{\text {MAX }}$ were applied to the cut samples. The crack propagated cycle by cycle and the extension of crack was recorded by a digital camera (Canon EOS5D) (Fig. 10a). We plotted the extension of crack $\Delta \mathrm{c}$ as a function of the number of cycles N (Fig. 10b-d). The crack propagated rapidly in the beginning and then reached a steady state afterwards. For most cases, after about 2000 cycles the extension of crack $\Delta c$ is almost a linear function of the number of cycles N. However, for a sample prepared using the solution of 3 M AAM and tested under the maximum stretch of $\lambda_{M A X}=1.6$, the crack propagation was observed after about 20 thousand of cycles and reached a steady state thereafter (Fig. 10d). If the maximum stretch of cyclic loads is small enough $\left(\lambda_{M A X}=1.5\right)$, the cracks of all the samples prepared using different AAM solutions propagated for a small number of cycles and then stayed stationary. Table 1 lists the data for all the samples tested for this paper.

The behavior of fatigue fracture of the cut samples is related to the shakedown of the uncut samples. For the uncut samples, the stresses are high in the beginning cycles and approach constant in the steady state after many cycles. Correspondingly, for the cut samples, the extension of crack per cycle is high in the beginning cycles and approaches constant in the steady state. The slope of the linear portion of the 
curve is the steady-state extension per cycle.

(a)

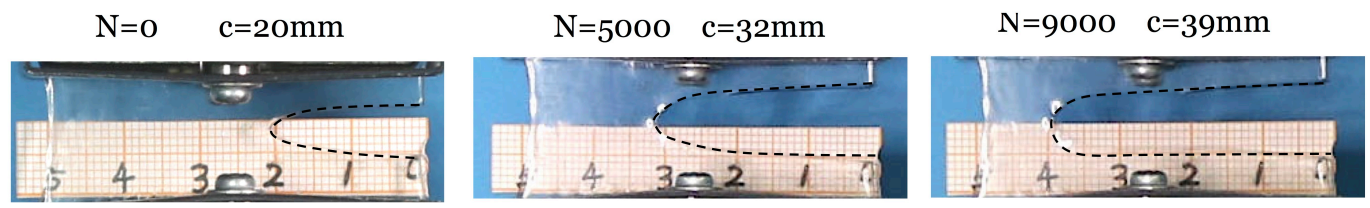

(b)

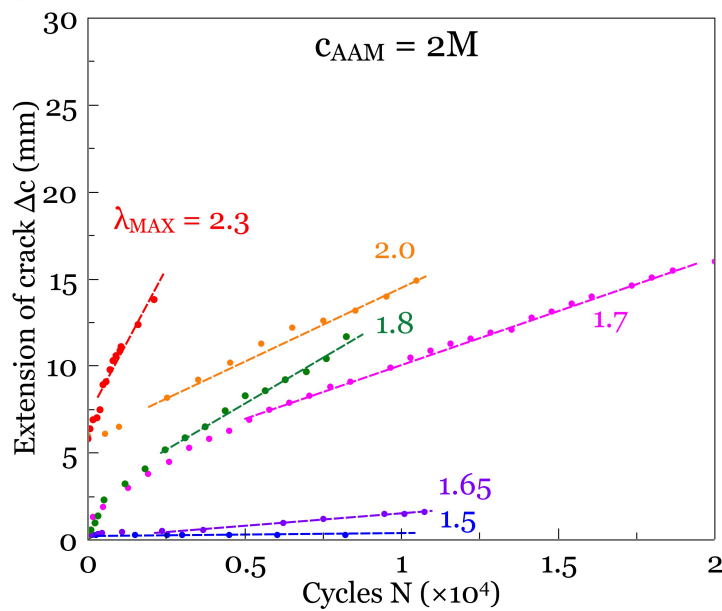

(d)

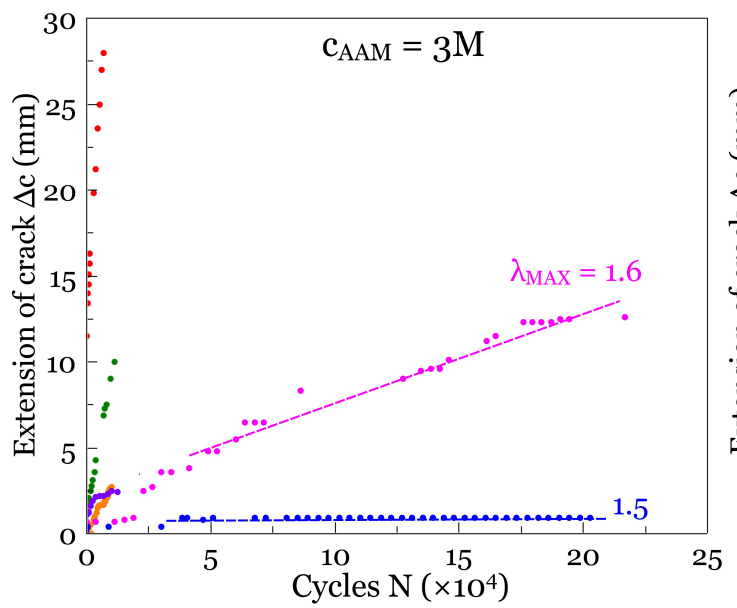

(c)

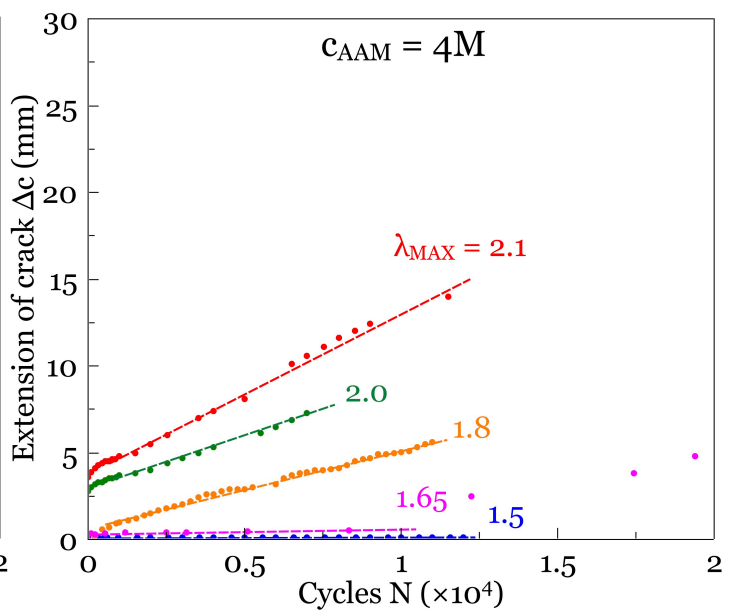

(e)

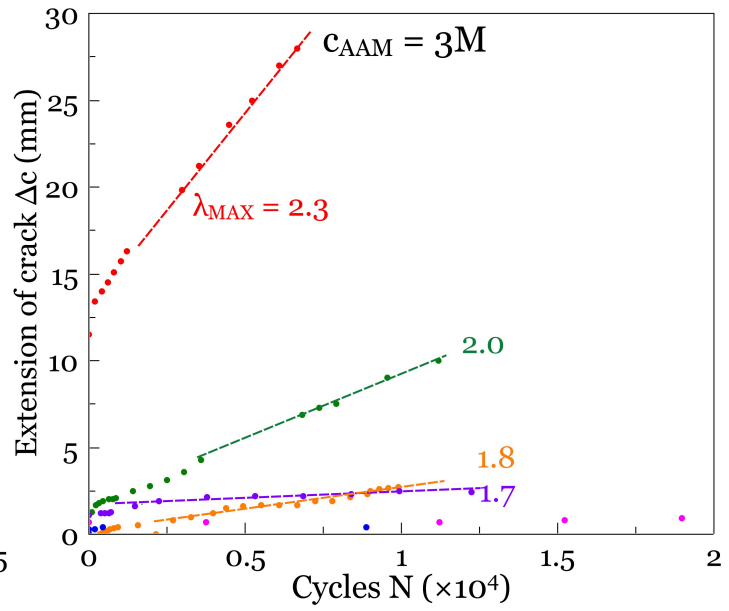

Fig. 10 Extension of crack under cyclic load. (a) The crack of a cut sample extends over cycles. (b)-(e) The extension of a crack $\Delta c$ is a function of the number of cycles $N$. The extension of a crack per cycle is large in the beginning cycles and then reaches a steady state afterwards. (d) and (e) show the same set of data in different ranges of cycles. 
Table1 Experimental data of the fatigue fracture tests

\begin{tabular}{|c|c|c|c|c|c|}
\hline $\begin{array}{c}\text { AAM } \\
\text { concentration } \\
\text { (M) }\end{array}$ & $\begin{array}{l}\text { maximum } \\
\text { stretch }\end{array}$ & $\begin{array}{c}\text { frequency } \\
(\mathrm{Hz})\end{array}$ & $\begin{array}{l}\text { number } \\
\text { of cycles }\end{array}$ & $\begin{array}{c}\text { energy } \\
\text { release rate } \\
\left(\mathrm{J} / \mathrm{m}^{2}\right)\end{array}$ & $\begin{array}{c}\text { extension of crack } \\
\text { per cycle } \\
(\mu \mathrm{m} / \text { cycle })\end{array}$ \\
\hline \multirow{6}{*}{2} & 1.50 & 0.40 & 9000 & 299 & 0.00 \\
\hline & 1.65 & 0.36 & 11000 & 490 & 0.12 \\
\hline & 1.70 & 0.36 & 20000 & 554 & 0.65 \\
\hline & 1.80 & 0.36 & 8000 & 560 & 1.12 \\
\hline & 2.00 & 0.36 & 11000 & 829 & 0.88 \\
\hline & 2.30 & 0.35 & 3000 & 920 & 2.98 \\
\hline \multirow{6}{*}{3} & 1.50 & 0.58 & 200000 & 343 & 0.00 \\
\hline & 1.60 & 0.51 & 210000 & 448 & 0.05 \\
\hline & 1.70 & 0.43 & 13000 & 488 & 0.04 \\
\hline & 1.80 & 0.38 & 10000 & 670 & 0.24 \\
\hline & 2.00 & 0.36 & 12000 & 824 & 0.80 \\
\hline & 2.30 & 0.34 & 7000 & 932 & 2.19 \\
\hline \multirow{5}{*}{4} & 1.50 & 0.40 & 11000 & 432 & 0.00 \\
\hline & 1.65 & 0.36 & 9000 & 573 & 0.02 \\
\hline & 1.80 & 0.38 & 11000 & 607 & 0.45 \\
\hline & 2.00 & 0.37 & 11000 & 887 & 0.61 \\
\hline & 2.10 & 0.36 & 7000 & 1159 & 0.95 \\
\hline
\end{tabular}

To compare the data of fatigue fracture of different materials, it is a common practice to plot the extension of crack per cycle, $d c / d N$, as a function of the energy release rate, $G$. The practice was first reported for elastomers by Thomas in 1958 [81]. A similar practice using the stress intensity factor was first reported for metals by Paris et al. in 1961 [90]. For metals, fatigue fracture is commonly studied when deformation is small, except for a small zone around the tip of the crack. Outside 
the small zone, the material is well described by the linear theory of elasticity, and the stress intensity factor is a measure of the applied load. In the linear theory of elasticity, for a crack in a thin sheet, the stress intensity factor $K$ relates to the energy release rate $G$ as $K=\sqrt{E G}$, where $E$ is Young's modulus. Thus, the energy release rate and the stress intensity factor are alternative, but equivalent, measures of the applied load.

For elastomers, fatigue fracture is commonly studied when deformation is large in the entire sample. Still, for a highly elastic elastomer, outside a small zone around the tip of the crack, the material is well described by the nonlinear theory of elasticity, and the energy release rate is a measure of the applied load.

Consider a crack in an elastic material, linearly or nonlinearly elastic. So long as the inelastic zone around the tip of the crack is small compared to the size of the sample, the energy release rate is a measure of the applied load. Near the crack tip, the only length scale is the distance $R$ of a material particle in the sample from the tip of the crack. From a dimensional consideration, the elastic energy per unit volume with the unit $\mathrm{J} / \mathrm{m}^{3}$ is proportional to the energy release rate with the unit $\mathrm{J} / \mathrm{m}^{2}$ by $G / R$. For tough hydrogels, the entire sample is inelastic during fracture. This large-scale inelasticity is evident in the pronounced stress-stretch hysteresis and subsequent shakedown. Because no where in the sample does the theory of elasticity apply, it is unclear if the energy release rate is an adequate measure of the applied load.

Still, it is desirable to use a parameter to compare different materials. 
Following previous studies of fatigue fracture of hydrogels [73, 74] and elastomers [81], we calculate an effective energy release rate using a procedure described below. We use the stress-stretch curve of the $2^{2000^{\text {th }}}$ cycle of the uncut samples, when the hydrogels approach the steady state (Fig. 9d-f). We integrate the loading part of the stress-stretch curve to calculate the energy density $W\left(\lambda_{M A X}\right)$. The effective energy release rate is $G=H W\left(\lambda_{\text {MAX }}\right)$. The steady-state extension of crack per cycle $d c / d N$ is plotted as a function of the effective energy release rate $G$ (Fig. 11).
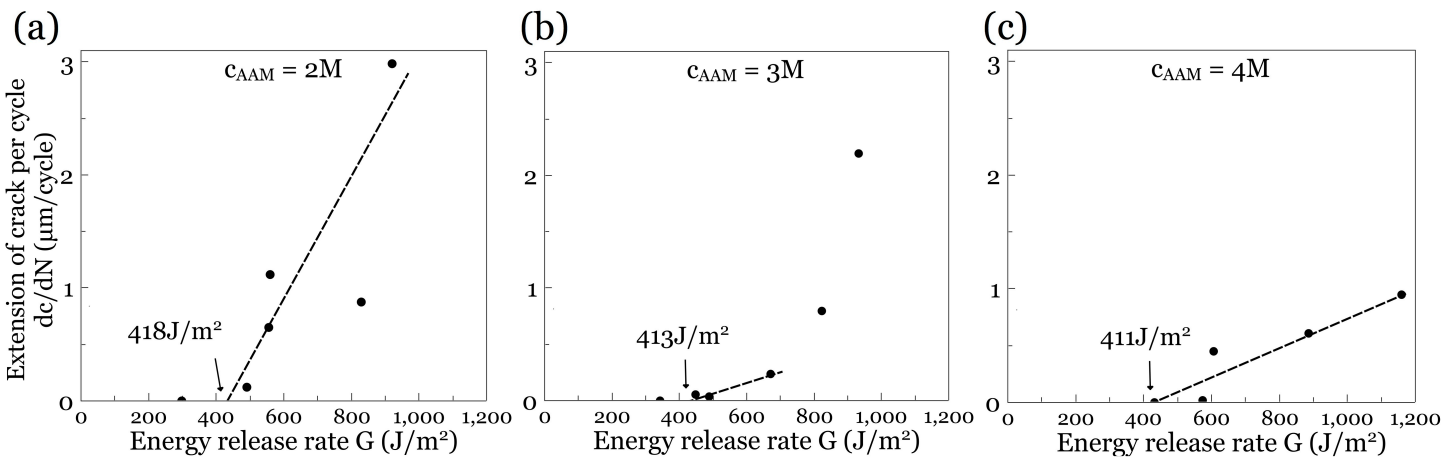

Fig. 11 The steady-state extension of crack per cycle is a function of the effective energy release rate for the DN hydrogels prepared using the solutions of three AAM concentrations. The intercept of a linear extrapolation gives the threshold of fatigue fracture.

In testing established materials, such as a commercial metallic alloy, a commonly adopted operational definition for fatigue threshold is the energy release rate at which the crack extends at a particular rate, say $10^{-11} \mathrm{~m} /$ cycle $[83,91]$. If the method used to measure crack length is accurate to $10^{-4} \mathrm{~m}$, and if the extension of the crack is undetected for $10^{7}$ cycles, a threshold is declared. This large number of cycles takes several months of nonstop testing at the rate of 1 second per cycle. The time is too 
long to be practical during the development of new materials. Instead, we adopt the following procedure to estimate threshold [74].

We linearly extrapolate the data points in the plane of $G$ and $d c / d N$, and take the intercept of the $G$ axis as the threshold of the fatigue fracture. In this linear extrapolation, we use the data close to the largest number of cycles tested, and ignore the data far away. This procedure gives the thresholds of fatigue fracture as $418 \mathrm{~J} / \mathrm{m}^{2}$ for the hydrogels prepared using the solution of 2 M AAM, $413 \mathrm{~J} / \mathrm{m}^{2}$ for $3 \mathrm{M}$ AAM, and $411 \mathrm{~J} / \mathrm{m}^{2}$ for $4 \mathrm{M}$ AAM. The threshold of fatigue fracture of DN hydrogels is about an order of magnitude lower than their toughness. Below the threshold, we did not detect the extension of crack within the numbers of cycles tested (Table 1).

\section{Prediction of threshold using the Lake-Thomas model}

The threshold of fatigue fracture in an elastomer is commonly interpreted in terms of the Lake-Thomas model [82]. The model assumes that, at a load approaching the threshold, the extension of a crack only activates a single dissipative process in the elastomer: breaking polymer chains ahead the crack. That is, the model assumes that the hysteresis in the bulk of the elastomer does not contribute to the threshold. The chemical bonds along each polymer chain are much stronger than the physical interactions between different polymer chains. Consequently, when a chain is pulled nearly to the breaking point, all the chemical bonds of the entire chain is pulled to the same state. When the chain breaks at a single bond, the energy in the entire chain dissipates. The Lake-Thomas model estimates the 
threshold by the chemical energy in a unit area of a single layer of chains.

Incidentally, the Lake-Thomas model is analogous to a lower-bound model for the threshold of fatigue fracture in a metal. In a metal, bonds between any two atoms are comparably strong, and the lower-bound model estimates the threshold by the energy needed to break a single layer of atomic bonds [50]. That is, the model assumes that the hysteresis in the bulk of the sample does not contribute to the threshold, and estimates the threshold by the Griffith limit: $\Gamma_{o}=\mathbf{2} \gamma$, where $\gamma$ is the surface energy. The surface energy of a metal is on the order of $1 \mathrm{~J} / \mathrm{m}^{2}$. This model provides a lower bound. The experimental values of the threshold for metals are in the range $10-1000 \mathrm{~J} / \mathrm{m}^{2}$.

The Lake-Thomas model has been adapted to a single-network PAAM hydrogel [73]. For the PAMPS/PAAM double-network hydrogels, we now hypothesize that the rupture of the PAMPS network does not contribute to the threshold, and estimate the threshold by the chemical energy in one layer of PAAM chains:

$$
\Gamma_{\mathrm{o}}=\phi_{P A A M}^{\frac{2}{3}} \sqrt{n} b U l
$$

where $\phi_{P A A M}$ is the volume fraction of the PAAM network in the DN hydrogel, $n$ is the number of monomer units in a PAAM chain, $b$ is the number of bonds per unit volume of the dry polymer, $U$ is C-C bond energy, and $l$ is the length of each monomer unit.

We use the values of $\phi_{P A A M}$ determined by the experiments described above. 
The number of single bonds per unit volume of the polymer is estimated by the number of monomers per unit volume of the dry polymer, $b=\frac{A \rho}{m}=1.12 \times 10^{28} \mathrm{~m}^{-3}$, where $m$ is the molecular weight of acrylamide $(71.08 \mathrm{~g} / \mathrm{mole}), \rho$ is the density of acrylamide $\left(1.322 \mathrm{~g} / \mathrm{cm}^{3}\right)$, and $A$ is the Avogadro number $\left(6.022 \times 10^{23}\right)$. The length of the monomer is estimated by $l=b^{-\frac{1}{3}}=0.446 \mathrm{~nm}$. The energy of a C-C bond is $U=3.3 \times 10^{-19} J$ [82]. The molar ratio of the crosslinker MBAA to monomer AAM is set to be $0.001 \mathrm{~mol} \%$, and we estimate the number of monomers between two crosslinks by $n=1 / 0.001 m o l \%=10^{5}$. The threshold of fatigue fracture for PAMPS/PAAM hydrogels prepared using the solution of $2 \mathrm{M}$ AAM is predicted to be $92 \mathrm{~J} / \mathrm{m}^{2}, 112 \mathrm{~J} / \mathrm{m}^{2}$ for $3 \mathrm{M}$ AAM, and $125 \mathrm{~J} / \mathrm{m}^{2}$ for $4 \mathrm{M}$ AAM.

\section{Effect of the length of PAAM chains.}

The Lake-Thomas model adapted to the DN hydrogel gives a prediction of correct order of magnitude. Here we do not push for better agreement. Instead, we ascertain if the threshold does change if we change the density of crosslinkers of the PAAM network. We prepared another set of samples of DN hydrogels. The formulation of the PAMPS network is the same as before. We immersed the PAMPS network in a solution of $2 \mathrm{M} \mathrm{AAM}$, with $0.01 \mathrm{~mol} \% \mathrm{MBAA}$ and $0.01 \mathrm{~mol} \%$ OA relative 
to AAM. That is, the density of the crosslinkers for the PAAM network is 10 times that used in the above experiments. In the fully swollen DN hydrogel, the volume fractions of PAMPS, PAAM, and water are respectively, 1.1, 7.5, and 91 vol\%. We repeated the same experiments for the samples of this composition (Fig. 12). The fracture energy and the fatigue threshold of this set of samples are $3066 \mathrm{~J} / \mathrm{m}^{2}$ and $220 \mathrm{~J} / \mathrm{m}^{2}$. Recall that the fracture energy and the fatigue threshold for the hydrogels using the solution of $2 \mathrm{M}$ AAM with $0.001 \mathrm{~mol} \% \mathrm{MBAA}$ is $3779 \mathrm{~J} / \mathrm{m}^{2}$ and $418 \mathrm{~J} / \mathrm{m}^{2}$. The Lake-Thomas model predicts that if the crosslinker density of PAAM increases by ten times, the number of monomers between two crosslinks decreases to one tenth, resulting in a decrease of threshold by a factor of $\sqrt{10}$. This prediction is captured in our experiments qualitatively, but not quantitatively.

"The number of monomers between two crosslinks is roughly $n=7000$ for both PAAM gel and alginate/PAAM gel in Ref [73] and [74], and is $n=10^{5}$ for DN gels prepared in the solution of $10^{-5}$ number fraction of MBAA relative to AAM in this work. The corresponding fatigue threshold is $7 \mathrm{~J} / \mathrm{m}^{2}$ for pure PAAM gel, $53 \mathrm{~J} / \mathrm{m}^{2}$ for alginate/PAAM gel, and $400 \mathrm{~J} / \mathrm{m}^{2}$ for $\mathrm{DN}$ gel. We can see that the comparisons of fatigue threshold from different gel systems do not simply support the assumption that the sacrificial network has no contribution. The detailed reasons are still unknown and are worthy of further investigations. 
(a)

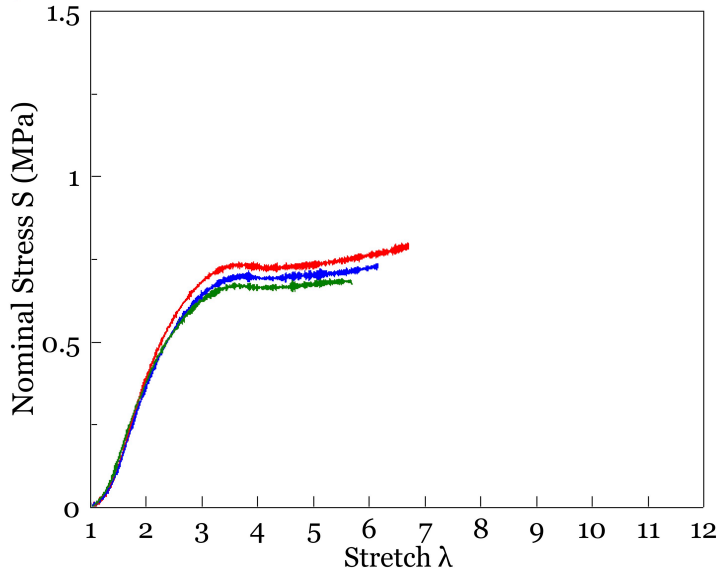

(c)

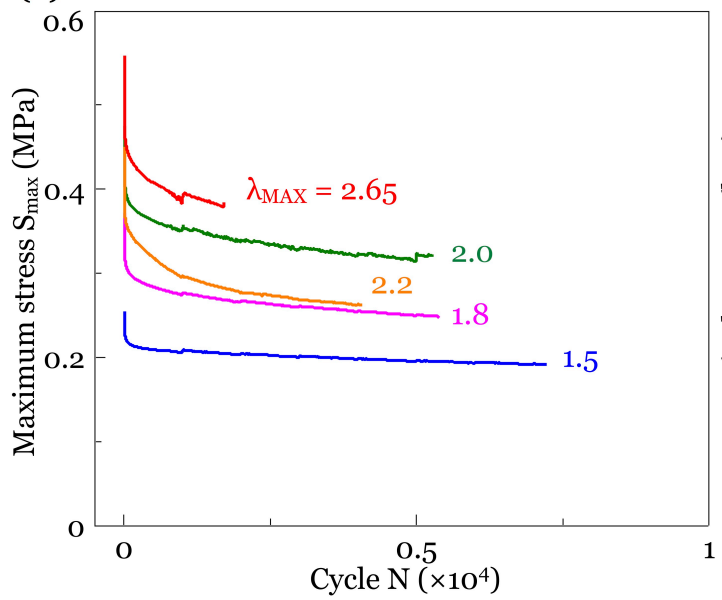

(e)

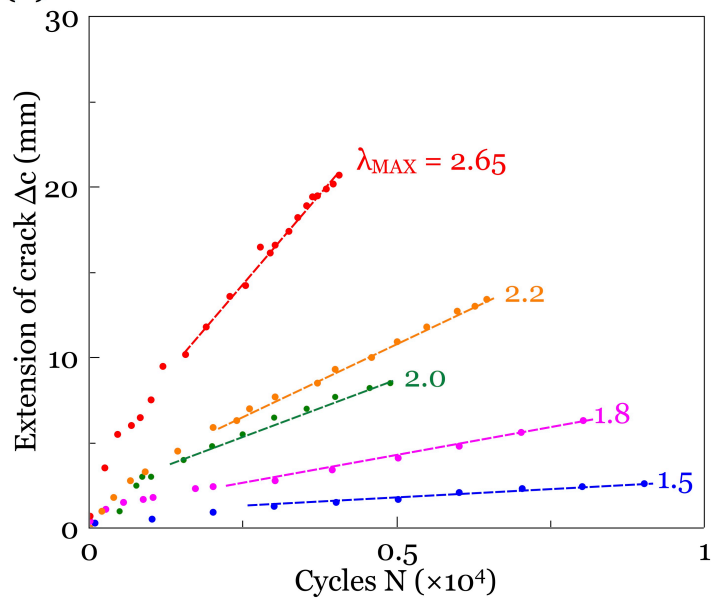

(b)

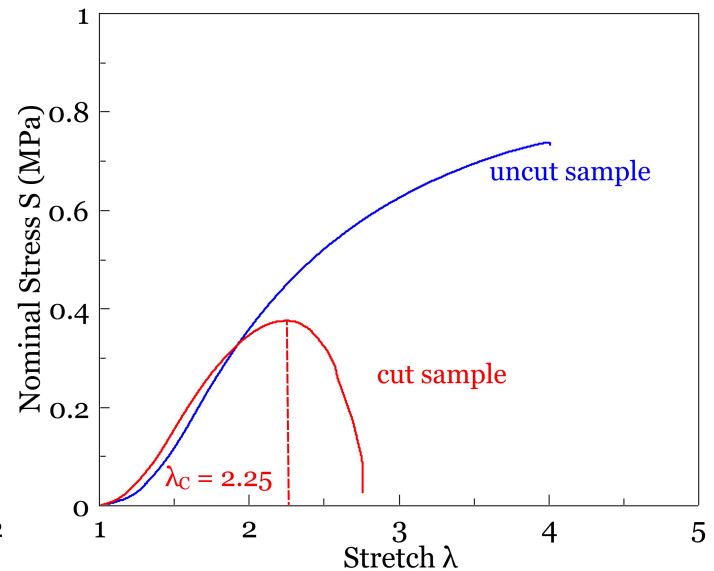

(d)

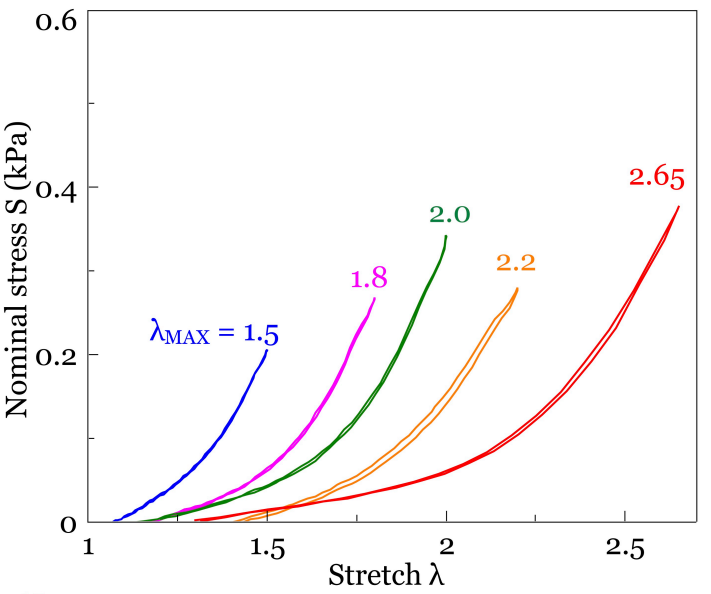

(f)

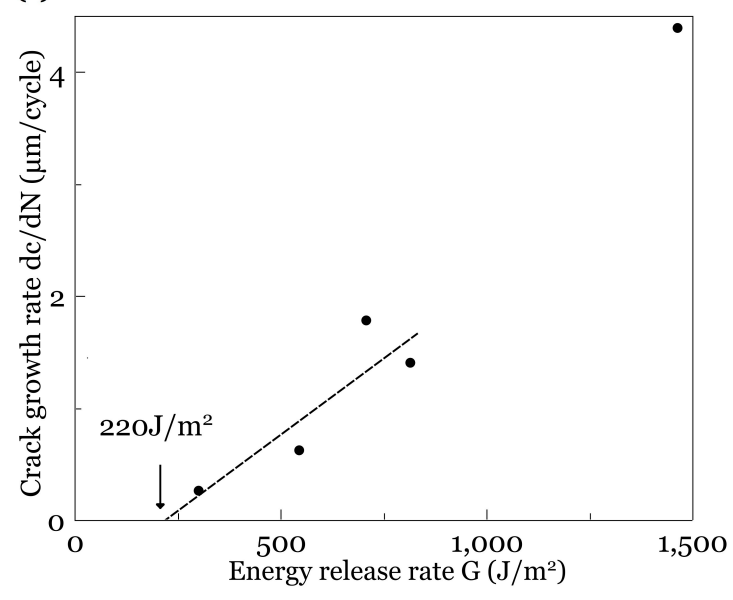

Figure 12 Data for DN hydrogels prepared with the solution of 2M AAM and 0.01 mol\% MBAA. (a) The stretch-stress curves determined by uniaxial tensile tests. (b) The stress-stretch curves of uncut and cut samples determined by pure shear tests. (c) Maximum stresses of uncut samples evolve over cycles. (d) Stress-stretch curves of uncut samples at the $\mathbf{2 0 0 0}^{\text {th }}$ cycle. (e) The extension of crack of cut samples under 
cyclic loads. (f) The steady-state extension of crack per cycle as a function of the energy release rate.

\section{Concluding remarks}

This paper is the first study of the fatigue of the classic PAMPS/PAAM double network hydrogels. We apply cyclic stretch to samples with or without cuts. For an uncut sample, damage accumulates over thousands of cycles, until a steady state is reached. For a cut sample, the crack extends cycle by cycle if the amplitude of the load is above a threshold. We prepare all samples of the DN hydrogels using PAMPS hydrogels of a fixed composition, but using aqueous solutions of various concentrations of AAM monomers and MBAA crosslinkers. Within the samples prepared for this work, the effect of the concentration of AAM is modest. The concentration of MBAA has a small effect on fracture energy, but significant effect on threshold energy. For the DN hydrogels prepared in the aqueous solution of $2 \mathrm{M}$ AAM and $10^{-5}$ number fraction of MBAA relative to AAM, the fracture energy is 3779 $\mathrm{J} / \mathrm{m}^{2}$, and the fatigue threshold is $418 \mathrm{~J} / \mathrm{m}^{2}$. For the DN hydrogels prepared in the aqueous solution of $2 \mathrm{M}$ AAM and $10^{-4}$ number fraction of MBAA relative to AAM, the fracture energy is $3066 \mathrm{~J} / \mathrm{m}^{2}$, and the fatigue threshold is $220 \mathrm{~J} / \mathrm{m}^{2}$.

We adapt the Lake-Thomas model under the hypothesis that the fatigue threshold of a DN hydrogel corresponds to the chemical energy stored in one layer of PAAM chains. This hypothesis predicts the experimentally measured fatigue thresholds qualitatively. The available data do not allow a comprehensive 
comparison of the fatigue-resistance of hydrogels of different chemistries, such as the alginate/PAAM (Fig. 4) and PAMPS/PAAM (Fig. 11), given that fatigue resistance depends on the concentrations of ingredients. It is hoped that researchers worldwide will report the fatigue behavior of their own hydrogels under development for load-bearing applications, and that an understanding will soon emerge to link the behavior of fatigue to the chemistry of hydrogels.

\section{Acknowledgments}

ZS acknowledges the support of the NSF MRSEC at Harvard (DMR-1420570), and the visiting appointment at Xian Jiaotong University. TL acknowledges the support of NSFC (11772249). JH acknowledges the support of NSFC (11702207). JT acknowledges the support of NSFC (11702208) and the Program for Postdoctoral Innovative Talents (No. BX201700192).

\section{References}

1. Buwalda SJ, Boere KWM, Dijkstra PJ, Feijien J, Vermonden T, Hennink WE. Hydrogels in a historical perspective: From simple networks to smart materials. J Control Release 2014; 190: 254-273.

2. Wichterle O, Lim D. Hydrophilic gels for biological use. Nature 1960; 185(4706): 117-118.

3. Masuda F. Trends in the development of superabsorbent polymers for diapers; 1994. 
4. Li J, Mooney DJ. Designing hydrogels for controlled drug delivery. Nat Rev Mater 2016; 1: 16071.

5. Zhang YS, Khademhosseini A. Advances in engineering hydrogels. Science 2017; 356(6337): eaaf3627.

6. Beebe DJ, Moore JS, Bauer JM, Yu Q, Liu RH, Devadoss C, Jo B-H. Functional hydrogel structures for autonomous flow control inside microfluidic channels. Nature 2000; 404(6778): 588.

7. Sidorenko A, Krupenkin T, Taylor A, Fratzl P, Aizenberg J. Reversible switching of hydrogel-actuated nanostructures into complex micropatterns. Science 2007; 315(5811): 487-490.

8. Keplinger C, Sun J-Y, Foo CC, Rothemund P, Whitesides GM, Suo Z. Stretchable, transparent, ionic conductors. Science 2013; 341(6149): 984-987.

9. Li T, Li G, Liang Y, Cheng T, Dai J, Yang X, Liu B, Zeng Z, Huang Z, Luo Y, Xie T, Yang W. Fast-moving soft electronic fish. Sci Adv 2017; 3(4): e1602045.

10. Sun J-Y, Keplinger C, Whitesides GM, Suo Z. Ionic skin. Adv Mater 2014; 26(45): $7608-7614$

11. Robinson SS, O’Brien KW, Zhao H, Peele BN, Larson CM, Mac Murray BC, Van Meerbeek IM, Dunham SN, Shepherd RF. Integrated soft sensors and elastomeric actuators for tactile machines with kinesthetic sense. Extreme Mech Lett 2015; 5: 47-53.

12. Wirthl D, Pichler R, Drack M, Kettlguber G, Moser R, Gerstmayr R, Hartmann F, Bradt E, Kaltseis R, Siket CM, Schausberger SE, Hild S, Bauer S, Kaltenbrunner 
M. Instant tough bonding of hydrogels for soft machines and electronics. Sci Adv 2017; 3(6): e1700053.

13. Yang CH, Chen B, Lu JJ, Yang JH, Zhou J, Chen YM, Suo Z. Ionic cable. Extreme Mech Lett 2015; 3: 59-65.

14. Kim C-C, Lee H-H, Oh KH, Sun J-Y. Highly stretchable, transparent ionic touch panel. Science 2016; 353(6300): 682-687.

15. Sarwar MS, Dobashi Y, Preston C, Wyss JKM, Mirabbasi S, Madden JDW. Bend, stretch, and touch: locating a finger on an actively deformed transparent sensor array. Sci Adv 2017; 3(3): e1602200.

16. Yang $\mathrm{CH}$, Chen B, Zhou J, Chen YM, Suo Z. Electroluminescence of giant stretchability. Adv Mater 2016; 28(22): 4480-4484.

17. Larson C, Peele B, Li S, Robinson S, Totaro M, Beccai L, Mazzolai B, Shepherd R. Highly stretchable electroluminescent skin for optical signaling and tactile sensing. Science 2016; 351(6277): 1071-1074.

18. Pu X, Liu M, Chen $\mathrm{X}$, Sun J, Du C, Zhang $\mathrm{Y}$, Zhai J, Hu W, Wang ZL. Ultrastretchable, transparent triboelectric nanogenerator as electronic skin for biomechanical energy harvesting and tactile sensing. Sci Adv 2017; 3(5): e1700015.

19. Yang CH, Zhou S, Shian S, Clarke DR, Suo Z. Organic liquid-crystal devices based on ionic conductors. Mater Horiz 2017;10.1039/C7MHoo345E. 
20. Bai Y, Chen B, Xiang F, Zhou J, Wang H, Suo Z. Transparent hydrogel with enhanced water retention capacity by introducing highly hydratable salt. Appl Phys Lett 2014; 105(15): 151903.

21. Yuk H, Zhang T, Parada GA, Liu X, Zhao X. Skin-inspired hydrogel-elastomer hybrids with robust interfaces and functional microstructures. Nat Commun 2016; 7: 12028.

22. Parada GA, Yuk H, Liu X, Hsieh AJ, Zhao X. Impermeable robust hydrogels via hybrid lamination. Adv Healthcare Mater 2017: 1700520.

23. Le Floch P, Yao X, Liu Q, Wang Z, Nian G, Sun Y, Jia Y, Suo Z. Wearable and washable conductors for active textiles. ACS Appl Mater Inter 2017; 9(30): 25542-25552.

24. Zhang Q, Wang $\mathrm{X}, \mathrm{Mu}$ Q, Liu $\mathrm{P}$, Jia S, Chen L, Zhang X, Wang K, Wei Y. Genipin-cross-linked silk sericin/poly (N-isopropylacrylamide) IPN hydrogels: color reaction between silk sericin and genipin, pore shape and thermo-responsibility. Mater Chem Phys 2015; 166: 133-143.

25. Gong JP, Katsuyama Y, Kurokawa T, Osada Y. Double-network hydrogels with extremely high mechanical strength. Adv Mater 2003; 15(14):1155-1158.

26. Gong JP. Why are double network hydrogels so tough? Soft Matter 2010; 6: 2583-2590.

27. Ahmed S, Nakajima T, Kurokawa T, Haque MA, Gong JP. Brittle-ductile transition of double network hydrogels: mechanical balance of two networks as the key factor. Polymer 2014; 55: 914-923. 
28. Gong JP. Materials both tough and soft. Science 2014; 344(6180): 161-162.

29. Na Y-H, Kurokaw T, Katsuyama Y, Tsukeshiba H, Gong JP, Osada Y, Okabe S, Karino T, Shibayama M. Structural characteristics of double network gels with extremely high mechanical strength. Macromolecules 2004; 37: 5370-5374.

30. Nakayama A, Kakugo A, Gong JP, Osada Y, Takai M, Erata T, Kawano S. High mechanical strength double-network hydrogel with bacterial cellulose. Adv Funct Mater 2004; 14(11): 1124-1128.

31. Tsukeshiba H, Huang M, Na YH, Kurokawa T, Kuwabara R, Tanaka Y, Furukawa H, Osada Y, Gong JP. Effect of polymer entanglement on the toughening of double network hydrogels. J Phys Chem B 2005; 109(34): 16304-16309.

32. Na Y-H, Tanaka Y, Kawauchi Y, Furukawa H, Sumiyoshi T, Gong JP, Osada Y. Necking phenomenon of double-network gels. Macromolecules 2006; 39(14): $4641-4645$.

33. Tirumala VR, Tominaga T, Lee S, Butler PD, Lin EK, Gong JP, Wu W-L. Molecular model for toughening in double-network hydrogels. J Phys Chem B 2008; 112(27): 8024-8031.

34. Nakajima T, Furukawa H, Tanaka Y, Kurokawa T, Osada Y, Gong JP. True chemical structure of double network hydrogels. Macromolecules 2009; 42(6): 2184-2189.

35. Liang S, Wu ZL, Hu J, Kurokawa T, Yu QM, Gong JP. Direct observation on the surface fracture of ultrathin film double-network hydrogels. Macromolecules 2009; 44(8): 3016-3020. 
36. Nakajima A, Kurokawa T, Ahmed S, Wu W-L, Gong JP. Characterization of internal fracture process of double network hydrogels under uniaxial elongation. Soft Matter 2012; 9(6): 1955-1966.

37. Matsuda T, Nakajima T, Fukuda Y, Hong W, Sakai T, Kurokawa T, Chung U, Gong JP. Yielding criteria of double network hydrogels. Macromolecules 2012; 49(5): 1865-1872.

38. Sun J-Y, Zhao X, IIIeperuma WRK, Chauhuri O, Oh KH, Mooney DJ, Vlassak JJ, Suo Z. Highly stretchable and tough hydrogels. Nature 2012; 489(7414): 133-136.

39. Haraguchi K, Takehisa T. Nanocomposite hydrogels: a unique organic-inorganic network structure with extraordinary mechanical, optical, and swelling/de-swelling properties. Adv Mater 2002; 14(16):1120.

40. Mredha MTI, Kitamura N, Nonoyama T, Wada S, Goto K, Zhang X, Nakajima T, Kurokawa T, Takagi Y, Yasuda K, Gong JP. Anisotropic tough double network hydrogel from fish collagen and its spontaneous in vivo bonding to bone. Biomaterials 2017; 132: 85-95.

41. Zhao X. Multi-scale multi-mechanism design of tough hydrogels: building dissipation into stretchy networks. Soft Matter 2014; 10: 672-687.

42. Peak CW, Wilker JJ, Schmidt G. A review on tough and sticky hydrogels. Colloid Polym Sci 2013; 291 (9): 2031-2047.

43. Haque MA, Kurokawa T, Kamita G, Gong JP. Lamellar bilayers as reversible sacrificial bonds to toughen hydrogel: hysteresis, self-recovery, fatigue resistance, and crack blunting. Macromolecules 2011; 44(22): 8916-8924. 
44. Sun TL, Kurokawa T, Kuroda S, Ihsan AB, Akasaki T, Sato K, Haque MA, Nakajima T, Gong JP. Physical hydrogels composed of polyampholytes demonstrate high toughness and viscoelasticity. Nat Mater 2013; 12(10): 932-937.

45. Wang W, Zhang Y, Liu W. Bioinspired fabrication of high strength hydrogels from non-covalent interactions. Prog Polym Sci 2017; 71: 1-25.

46. Zhou X, Guo B, Zhang L, Hu G. Progress in bio-inspired sacrificial bonds in artificial polymeric materials. Chem Soc Rev 2017; DOI: 10.1039/c7csoo276a.

47. Chen Q, Chen H, Zhu L, Zheng J. Engineering of tough double network hydrogels. Macromol Chem Phys 2016; 217(9): 1022-1036.

48. Li J, Illeperuma WRK, Suo Z, Vlassak JJ. Hybrid hydrogels with extremely high stiffness and toughness. ACS Macro Lett 2014; 3(6): 520-523.

49. Yuk H, Zhang T, Lin S, Parada GA, Zhao X. Tough bonding of hydrogels to diverse nonporous surfaces. Nat Mater 2016; 15(2): 190.

50. Fleck NA, Muller GM, Ashby MF, Hutchinson JW. Strain gradient plasticity: theory and experiment. Acta Metall Mater 1994; 42(2): 475-487.

51. Liao I, Moutos FT, Estes BT, Zhao X, Guilak F. Composite three-dimensional woven scaffolds with interpenetrating network hydrogels to create functional synthetic articular cartilage. Adv Funct Mater 2013; 23(47): 5833-5839.

52. Illeperuma WRK, Rothemund $\mathrm{P}$, Suo Z, Vlassak JJ. Fire-resistant hydrogel-fabric laminates: a simple concept that may save lives. ACS Appl Mater Inter 2016; 8(3): 2071-2077. 
53. Huang Y, King DR, Sun TL, Nonoyama T, Kurokawa T, Nakajima T, Gong JP. Energy-dissipative matrices enable synergistic toughening in fiber reinforced soft composites. Adv Funct Mater 2017; 27(9): e1605350.

54. Irwin GR. Fracture dynamics. Fracturing of Metals 1948; 152.

55. Orowan E. Fracture and strength of solids. Rep Prog Phys 1949; 12(1): 185.

56. Andrews EH. Rupture propagation in hysteresial materials: stress at a notch. J Mech Phys Solids 1963; 11(4): 231-242.

57. Ducrot E, Chen Y, Bulters M, Sijbesma RP, Creto C. Toughening elastomers with sacrificial bonds and watching them break. Science 2014; 344(6180): 186-189.

58. McMeeking RM, Evans AG. Mechanics of transformation-toughening in brittle materials. J Am Ceram Soc 1982; 65(5): 242-246.

59. Evans AG. Perspective on the development of high-toughness ceramics. J Am Ceram Soc 1990; 73(2): 187-206.

6o. Du J, Thouless MD, Yee AF. Development of a process zone in rubber-modified epoxy polymers. Int J Fracture 1998; 92(3): 271-286.

61. Brown HR. A model of the fracture of double network gels. Macromolecules 2007; 40(10): 3815-3818.

62. Tanaka Y. A local damage model for anomalous high toughness of double-network gels. Europhys Lett 2007; 78(5): 56005 .

63. Fukui T, Kitamura N, Kurokawa T, Yokota M, Kondo E, Gong JP, Yasuda K. Intra-articular administration of hyaluronic acid increases the volume of the 
hyaline cartilage regenerated in a large osteochondral defect by implantation of a double-network gel. J Mater Sci: Mater Med 2014; 25(4): 1173-1182.

64. Arakaki K, Kitamura N, Fujiki H, Kurokawa T, Iwamoto M, Ueno M, Kanaya F, Osada Y, Gong JP, Yasuda T. Artificial cartilage made from a novel doublenetwork hydrogel: in vivo effects on the normal cartilage and ex vivo evaluation of the friction property. J Biomed Mater Res A 2010; 93(3): 1160-1168.

65. Azuma C, Yasuda K, Tanabe Y, Taniguro H, Kanaya F, Nakayama A, Chen YM, Gong JP, Osada Y. Biodegradation of high-toughness double network hydrogels as potential materials for artificial cartilage. J Biomed Mater Res A 2007; 81(2): 373-380.

66. Yasuda K, Kitamura N, Gong JP, Arakaki K, Kwon HJ, Onodera S, Chen YM, Kurokawa T, Kanaya F, Ohmiya Y, Osada Y. A novel double-network hydrogel induces spontaneous articular cartilage regeneration in vivo in a Large osteochondral defect. Macromol Biosci 2009; 9(4): 307-316.

67. Li J, Celiz AD, Yang J, Yang Q, Wamala I, Whyte W, Seo BR, Vasilyev NV, Vlassak JJ, Suo Z, Mooney DJ. Tough adhesives for diverse wet surfaces. Science 2017; 357(6349): 378-381.

68. Liu J, Pang Y, Zhang S, Cleveland C, Yin X, Booth L, Lin J, Lee YL, Mazdiyasni H, Saxton S, Kirtane AR, Von Erlach T, Rogner J, Langer R, Traverso G. Triggerable tough hydrogels for gastric resident dosage forms. Nat Commun 2017; 8:124.

69. Illeperuma WRK, Sun J, Suo Z, Vlassaka JJ. Fiber-reinforced tough hydrogels. Extreme Mech Lett 2014; 1: 90-96. 
70. Yuk H, Lin S, Ma C, Takaffoli M, Fang NX, Zhao X. Hydraulic hydrogel actuators and robots optically and sonically camouflaged in water. Nat Commun 2017; 8: 14230.

71. Zheng WJ, An N, Yang JH, Zhou J, Chen YM. Tough al-alginate/poly (n-isopropylacrylamide) hydrogel with tunable lcst for soft robotics. ACS Appl Mater Inter 2015; 7(3): 1758-1764.

72. Webber RE, Creton C, Brown HR, Gong JP. Large strain hysteresis and mullins effect of tough double-network hydrogels. Macromolecules 2007; 40(8): 2919-2927.

73. Tang J, Li J, Vlassak JJ, Z.Suo. Fatigue fracture of hydrogels. Extreme Mech Lett 2017; 10: 24-31.

74. Bai RB, Yang Q, Tang J, Morelle XP, Vlassak J, Suo Z. Fatigue fracture of tough hydrogels. Extreme Mech Lett 2017; 15: 91-96.

75. Joshia A, Fussellb G, Thomasa J, Hsuanb A, Lowmanb A, Kardunac A, Vresilovicd E, Marcolongoa M. Functional compressive mechanics of a PVA/PVP nucleus pulposus replacement. Biomaterials 2006; 27(2): 176-184.

76. Bai T, Zhang P, Han Y, Liu Y, Liu W, Zhao X, Lu W. Construction of an ultrahigh strength hydrogel with excellent fatigue resistance based on strong dipole-dipole interaction. Soft Matter 2011; 7(6): 2825-2831.

77. Yu Q, Bauer JM, Moore JS. Responsive biomimetic hydrogel valve for microfluidics. Appl Phys Lett 2001; 78(17): 2589-2591. 
78. Treloar LRG, Montgomery DJ. The physics of rubber elasticity. Phys Today 1959; 12: 32 .

79. Rivlin RS, Thomas AG. Rupture of rubber. I. Characteristic energy for tearing. J Polym Sci 1953; 10(3): 291-318.

80. Mars WV, Fatemi A. Factors that affect the fatigue life of rubber: a literature survey. Rubber Chem Technol 2004; 77(3): 391-412.

81. Thomas AG. Rupture of rubber. V. Cut growth in natural rubber vulcanizates. J Polym Sci 1958; 31(123): 467-480.

82. Lake GJ, Thomas AG. The strength of highly elastic materials. Proc R Soc Lond Ser A: Math Phys Sci 1967; 300(1460): 108-119.

83. Suresh S. Fatigue of materials. Cambridge University Press; 1998.

84. Münster S, Jawerth LM, Leslie BA, Weitz JI, Fabry B, Weitz DA. Strain history dependence of the nonlinear stress response of fibrin and collagen networks. $\mathrm{P}$ Natl Acad Sci 2013; 110(30): 12197-12202.

85. Belytschko T. Plane stress shakedown analysis by finite elements. Int J Mech Sci 1972; 14(9): 619-625.

86. Mackenzie D, Boyle JT, Hamilton R. The elastic compensation method for limit and shakedown analysis: a review. J Strain Anal Eng 2000; 35(3): 171-188.

87. Ceradini G. Dynamic shakedown in elastic-plastic bodies. J Eng Mech Div 1980; 106(3): 481-499.

88. Sawczuk A. Shakedown analysis of elastic-plastic structures. Nucl Eng Des 1974; 28(1): 121-136. 
89. Chinh PD. On shakedown theory for elastic-plastic materials and extensions. J Mech Phys Solids 2008; 56(5): 1905-1915.

90. Paris PC, Gomez MP, Anderson WE. A rational analytic theory of fatigue. Trend Eng 1961; 13(1): 9-14.

91. Ritchie RO. Near-threshold fatigue-crack propagation in steels. Int Met Rev 1979; 24(1): 205-230. 


\section{Appendix}

(a)
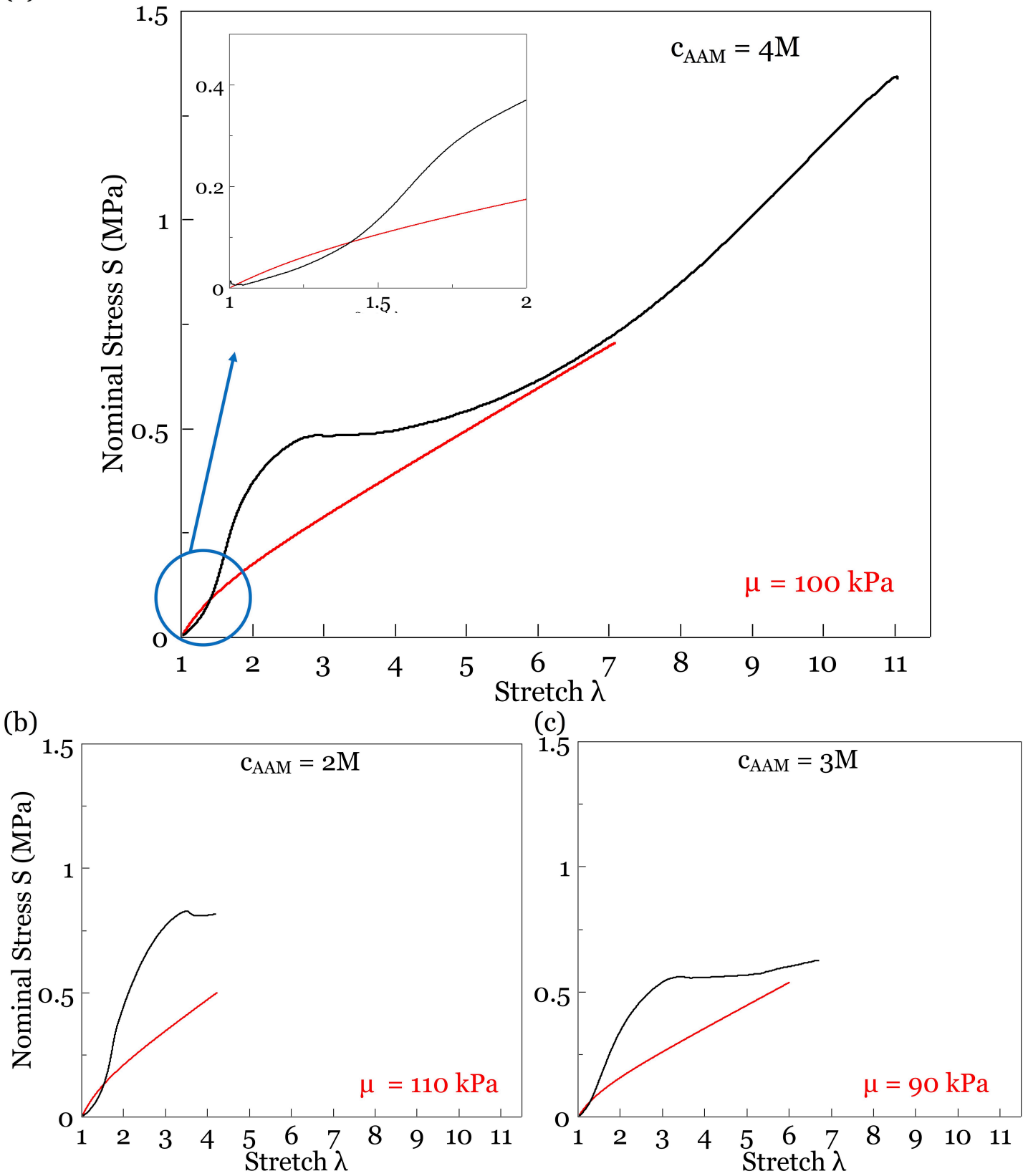

Fig. A1 Fit the beginning part of uniaxial stress-stretch curve by Eq. (2) with neo-Hookean model 
(a)

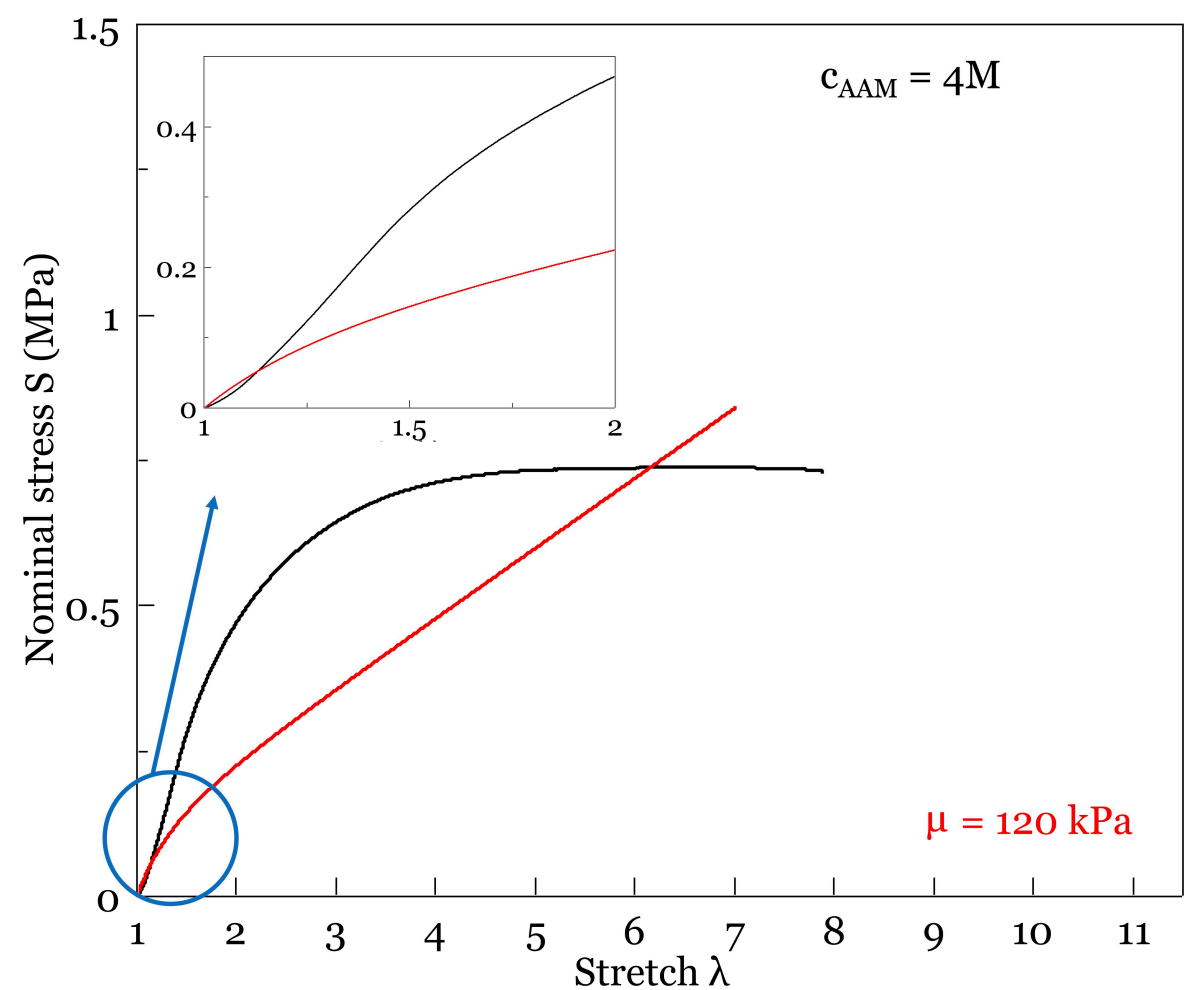

(b)

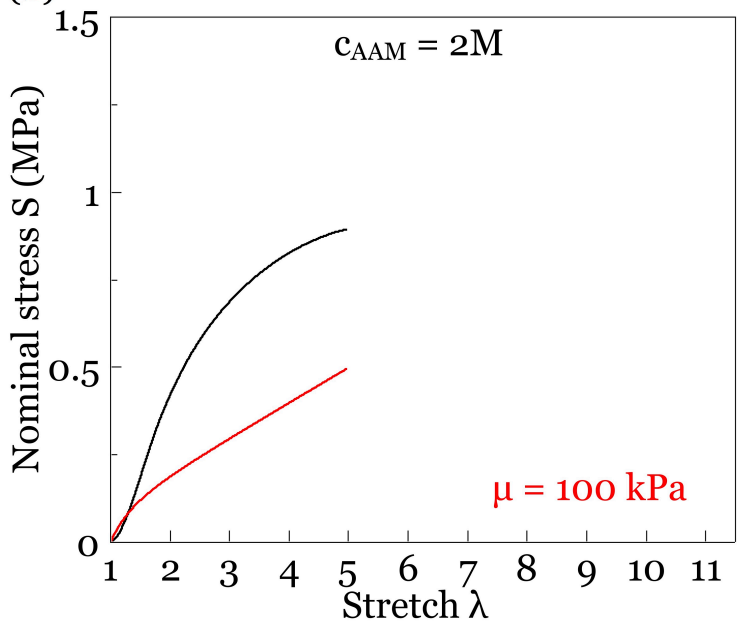

(c)

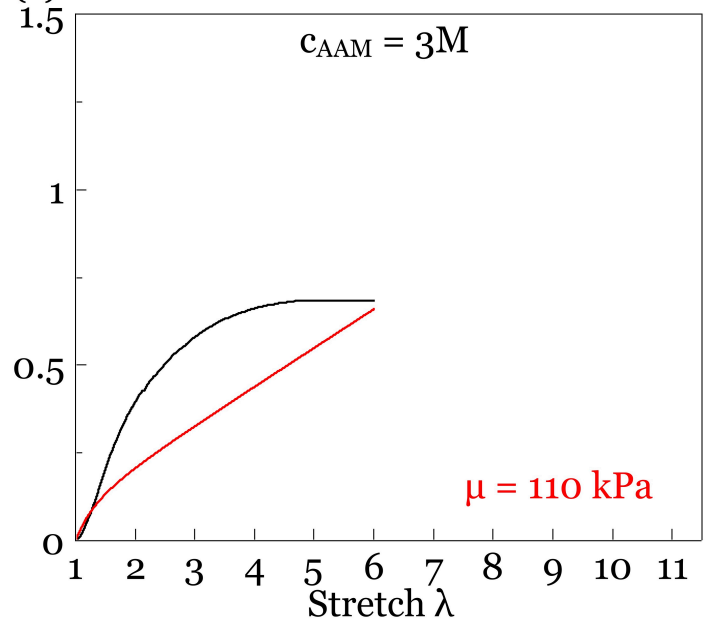

Fig. A2 Fit the beginning part of pure shear stress-stretch curve by Eq. (3) with neo-Hookean model 
(a)

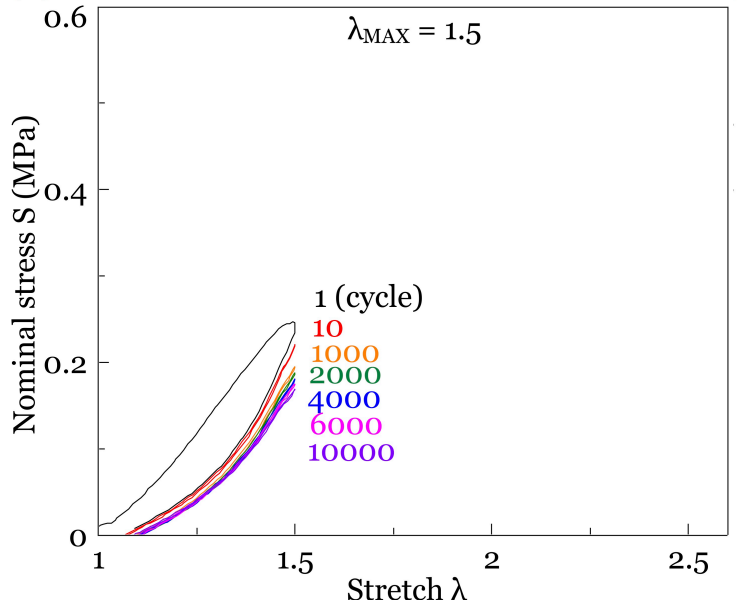

(c)

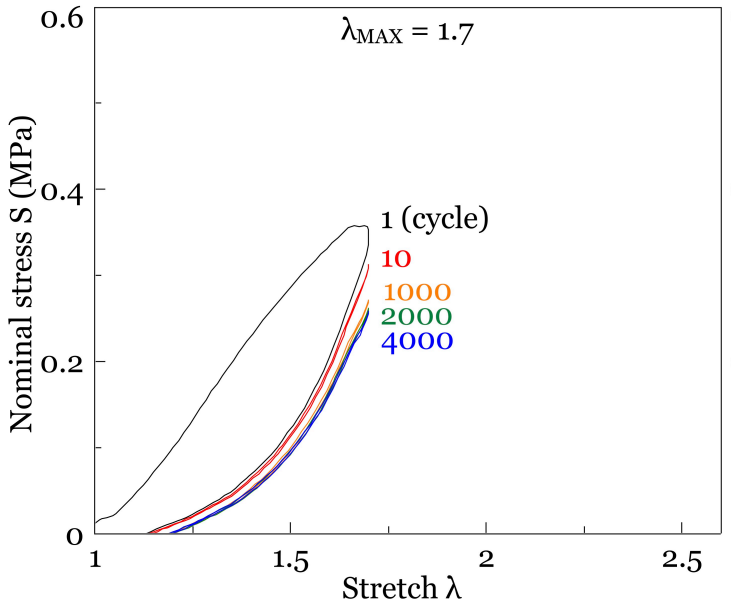

(e)

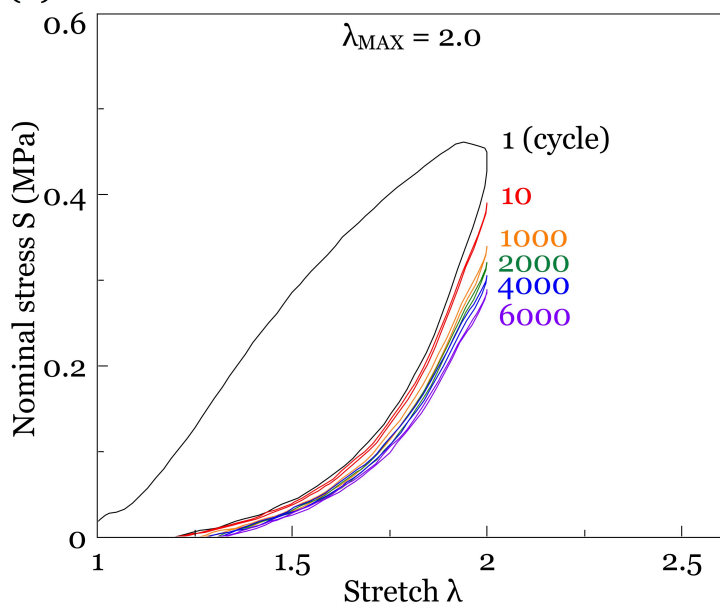

(b)

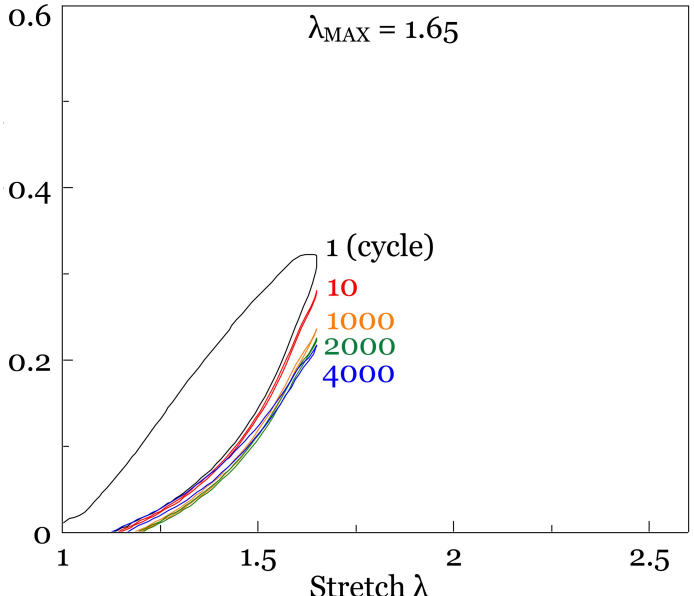

(d)

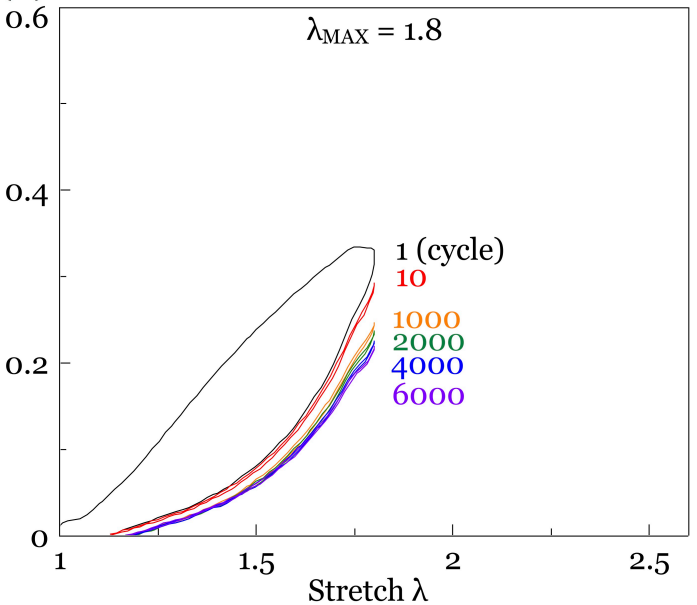

(f)

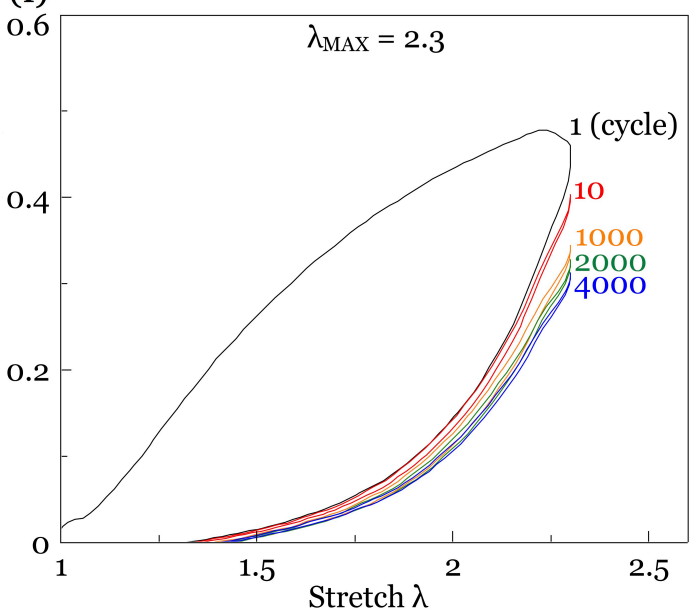

Fig. A3 Uncut samples shake down over thousands of cycles. The samples are prepared using the solution of 2 M AAM. 
(a)

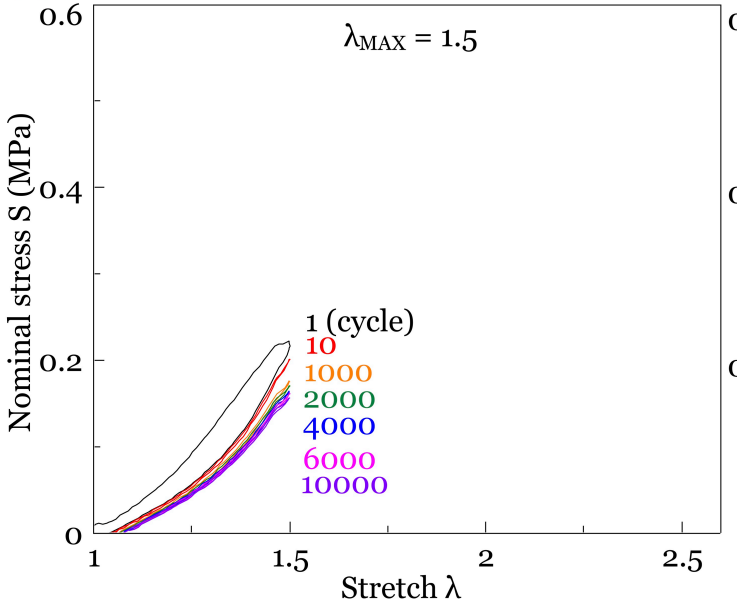

(c)

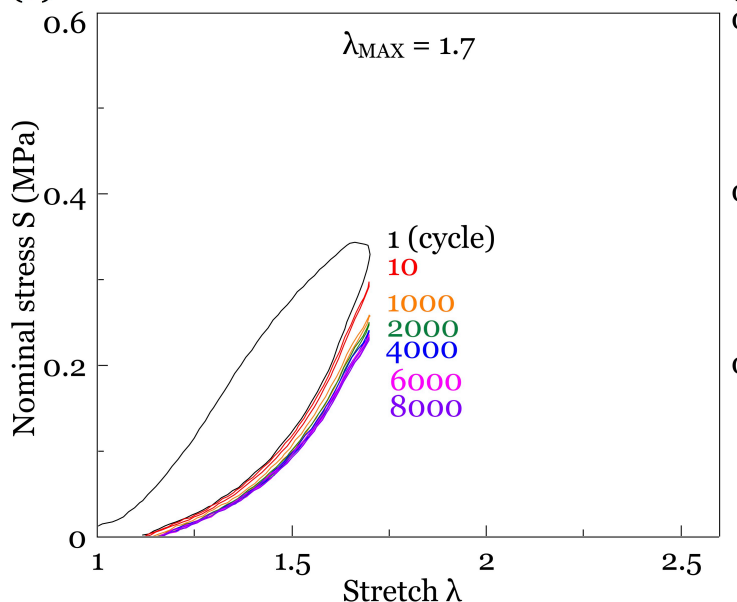

(e)

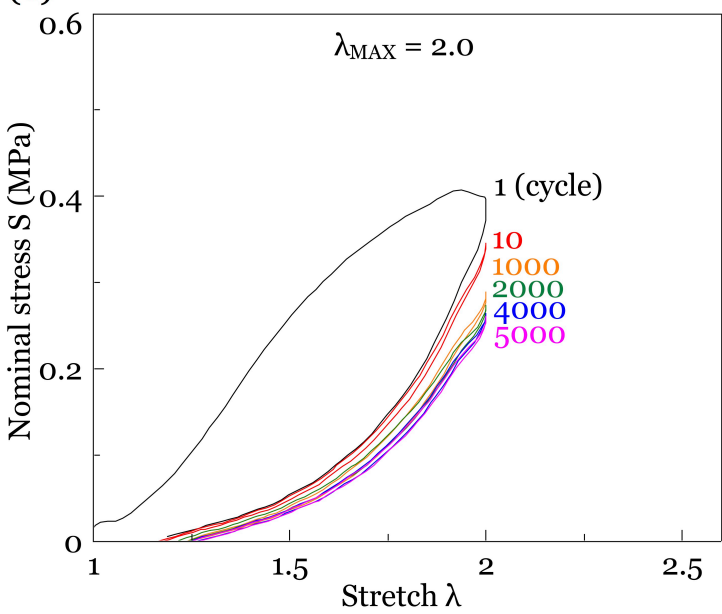

(b)

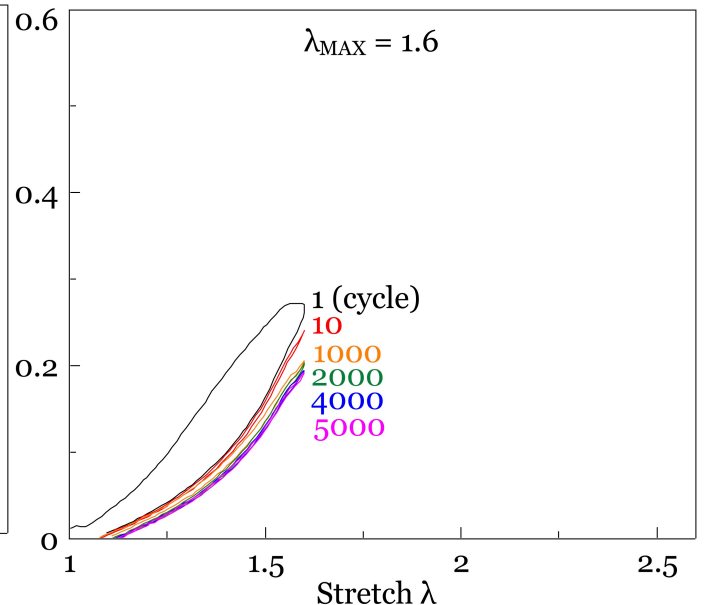

(d)

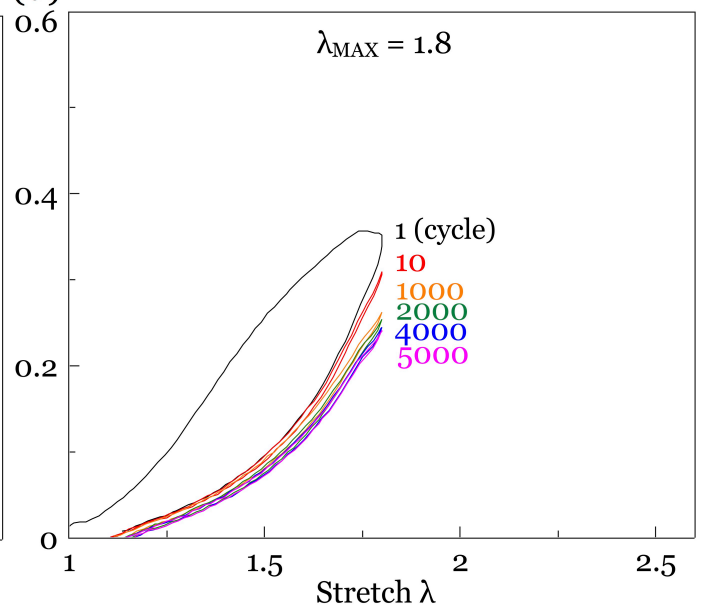

(f)

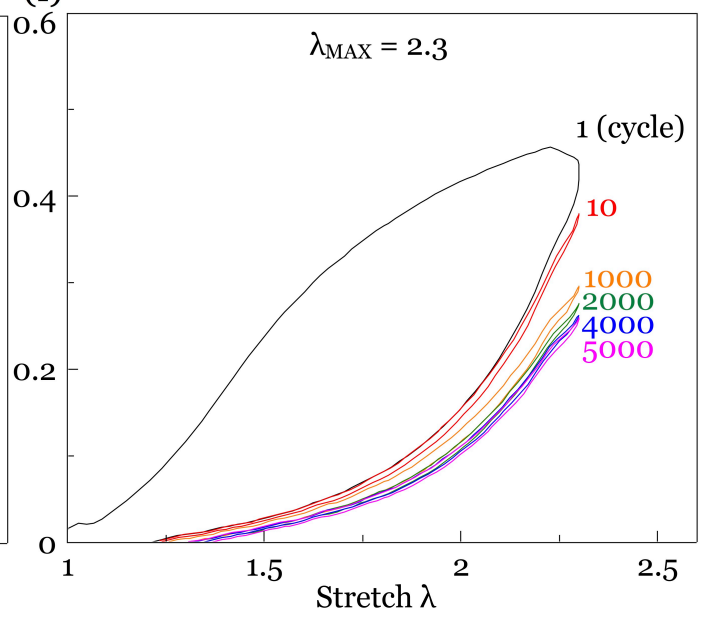

Fig. A4 Uncut samples shake down over thousands of cycles. The samples are prepared using the solution of 3 M AAM. 